
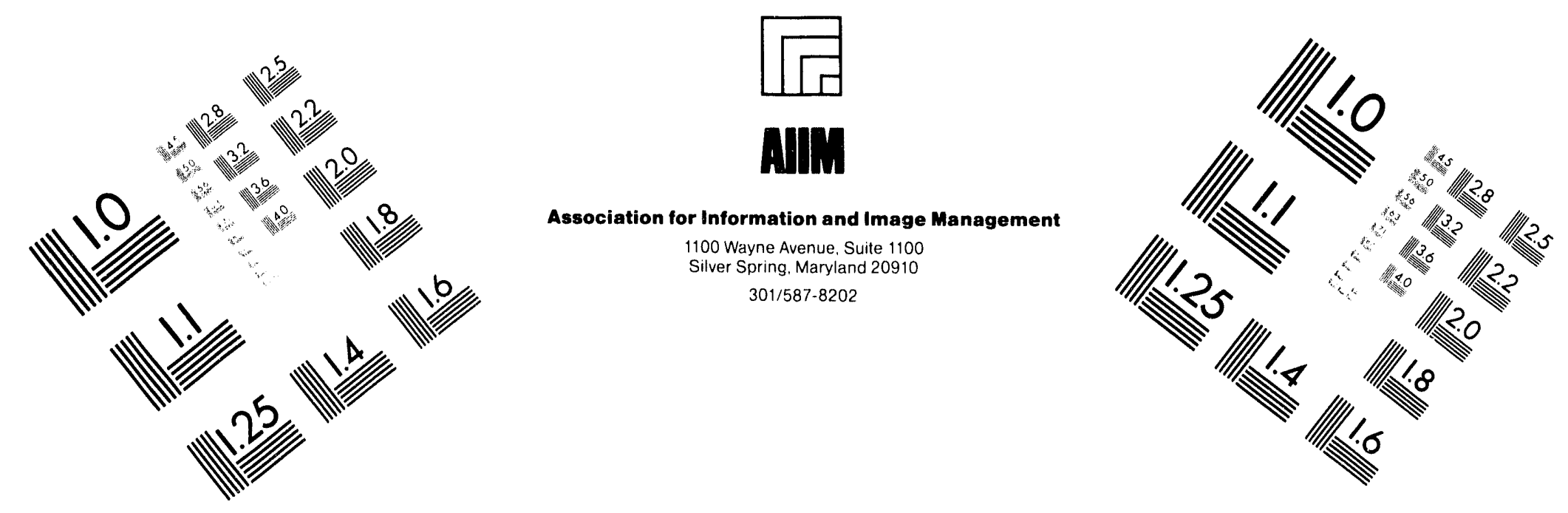

\title{
Centimeter
}

|

Inches
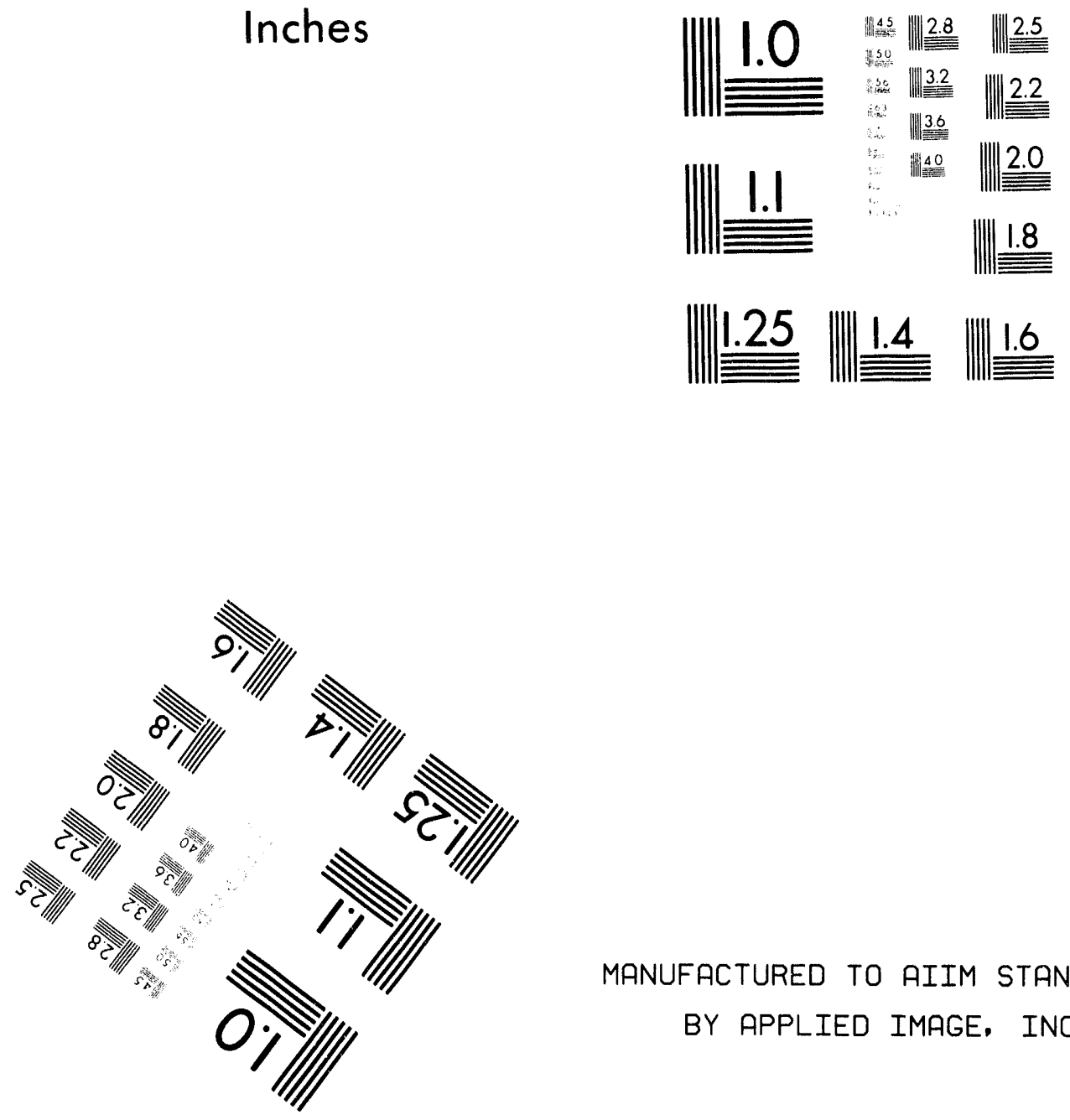

MANUFACTURED TO AIIM STANDARDS BY APPLIED IMAGE, INC.

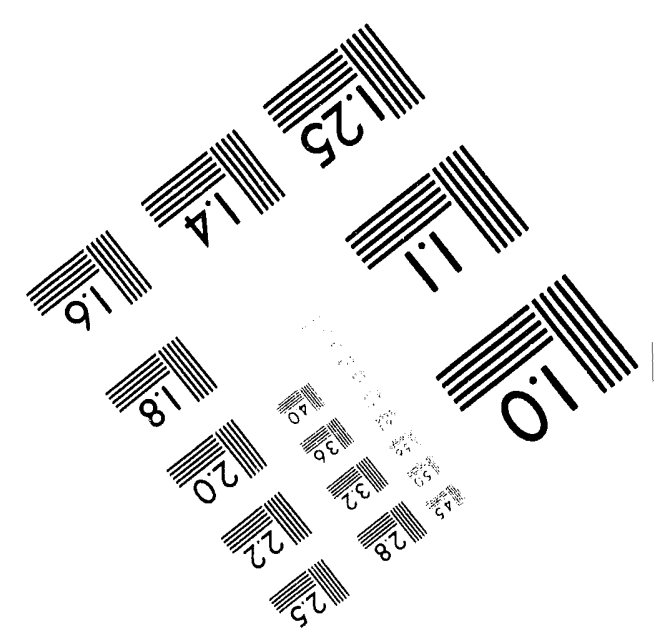



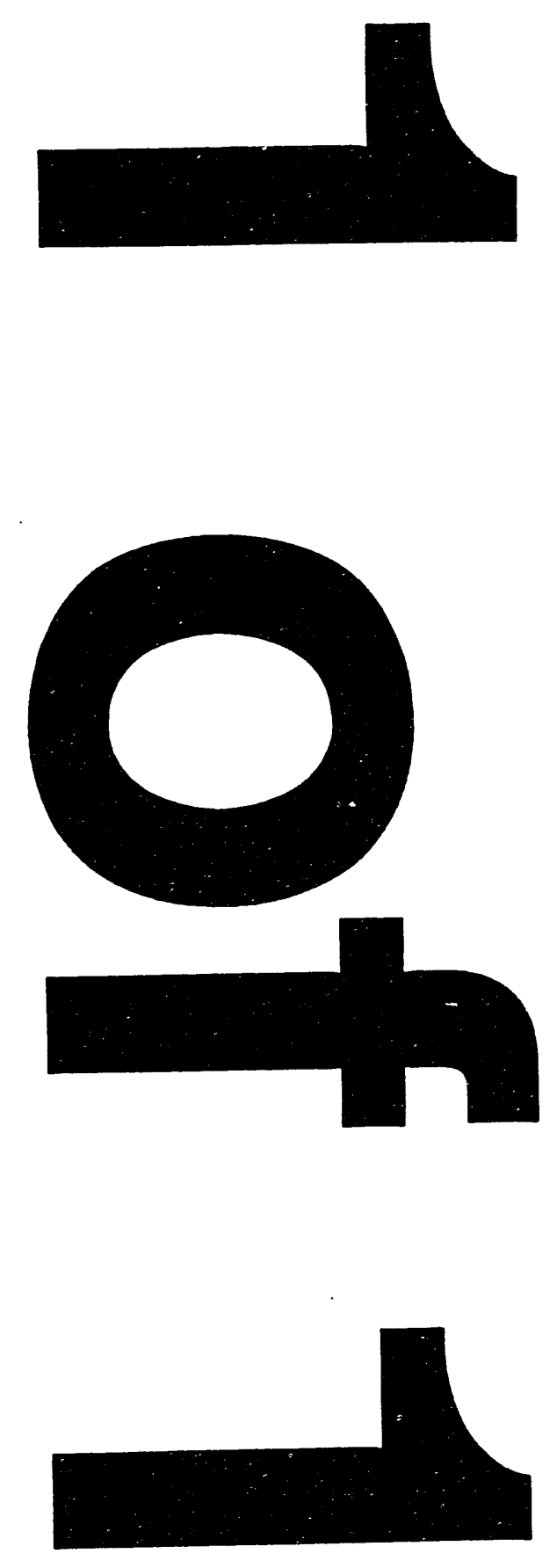
BNL-60557

UC-414

AGS/AD/94-4

INFORMAL

\section{ON SOLUTIONS OF SACHERER INTEGRAL EQUATION}

S.Y. Zhang and W.T. Weng

June 17,1994

ALTERNATING GRADIENT SYNCHROTRON DEPARTMENT

BROOKHAVEN NATIONAL LABORATORY

ASSOCIATED UNIVERSITIES, INC.

UPTON, LONG ISLAND, NEW YORK

UNDER CONTRACT NO. DE-AC02-76CH00016 WITH THE UNITED STATES DEPARTMENT OF ENERGY 


\section{DISCLAIMER}

This report was prepared as an account of work sponsored by the United States Government. Neither the United States nor the United States Department of Energy, nor any of their employees, nor any of their contractors, subcontractors, or their employees, makes any warranty, express or implied, or assumes any legal liability or responsibility for the accuracy, completeness, or usefulness of any information, apparatus, product or process disclosed, or represents that its use would not infringe privately owned rights. 


\title{
ON SOLUTIONS OF SACHERER INTEGRAL EQUATION *
}

\author{
S.Y. Zhang and W.T. Weng \\ AGS Department, Brookhaven National Laboratory, \\ Upton, New York 11973
}

\begin{abstract}
There are basically two approaches in solving the Sacherer integral equation, one uses the orthogonal polynomial expansion for radial functions, and another uses the Hankel harmonic samplings, both have extensive application. In this article, we discuss these approaches and present the corresponding solutions. If the azimuthal modes are not coupled, the Sacherer integral equation can be treated as an eigenvalue problem. It is shown that the information provided by the eigenvalue alone are insufficient in determining the system response. The initial perturbation pattern with respect to the radial modes must be considered. Several particle distributions are used as examples to show the solutions to the equation, and the physical implication of the radial modes is illustrated by using the examples.
\end{abstract}

* Work performed under the auspices of the U.S. Department of Energy 


\section{Introduction}

The coherent bunched beam instabiity can be described by the Sacherer integral equation 1 , developed from the Vlasov equllition on the particle evolution in phase space given impedances from the environment. The Sacherer integral equation (SIE) nowadays also becomes a foundation for further analysis of complicated beam dynamics, such as the microwave instability, the bunch lengthening, and various kinds of mode couplings $\{2,3 \mid$.

If the azimuthal modes are not coupled, such as in a low intensity regime, then the SIE can be reduced to an eigenvalue problem. where the two unknown variables, i.e. the coherent frequency shift and the radial function can be solved separately. There are basically two approaches in solving the SIE, and both have extensive applications. One is using the orthogonal polynomial expansion for radial functions $[4,5]$, and another is using Hankel harmonic samplings for radial functions $|3|$ !

The approach using orthogonal polynomials is conventional in treating problems such as the Sacherer integral equation. By using the lowest $\bar{k}$ orthogonal polynomials for the radial mode expansion, the SIE is transformed in to a $\bar{k}$ th order linear system. To determine the stability of the system, usually the eigenvalues should be found. However, in determining the system response, the information of eigenvalues alone is insufficient, the initial perturbation with respect to the radial modes must be included. In an extreme case, some eigenvalues, including unstable ones, may be virtually ineffective, in coupling to the initial perturbation.

In this article, we study the solutions for the Sacherer integral equation. We start from the orthogonal polynomial approach, and concentrate on the issues related to the radial modes. Using only necessary analytic mathematical means, the equation will be solved for several particle distributions, and the physical implications related to the radial modes are presented. The approach using Hankel harmonic samplings is also studied, and the relation and comparison of the two approaches are discussed. 


\section{Sacherer Integral Equation}

Consider the Vlasov equation,

$$
\frac{\partial \psi}{\partial t}+\phi \frac{\partial \psi}{\partial \phi}+\phi \frac{\partial \psi}{\partial \phi}=0
$$

where $\psi(\phi, \phi, t)$ is the normalized phase space density, and $\phi$ is the phase deviation of the particle. Using the phase space co-ordinates $\left(\phi, \phi / \omega_{S}\right)$ and the polar phase space coordinates,

$$
\begin{gathered}
\phi=r \cos \theta \\
\phi / \omega_{S}=r \sin \theta
\end{gathered}
$$

the equation (2-1) can be written as,

$$
\frac{\partial \psi}{\partial t}-\omega_{S} \frac{\partial \psi}{\partial \theta}+\left(\phi+\omega_{\tilde{S}}^{2} \phi\right) \frac{\partial \psi}{\partial \phi}=0
$$

The phase space density can be seen as a large stationary, or time independent part, $\psi_{0}$ and a small perturbation part $\psi_{p}$, which oscillates with the frequency $\omega$,

$$
\psi(r, \theta, t)=\psi_{0}(r, \theta)+\psi_{p}(r, \theta) e^{j \omega t}
$$

We note that in $(2-4)$, the term $\phi+\omega_{S}^{2} \phi$ represents a first order quantity, therefore $\frac{\partial \psi}{\partial \phi}$ can be replaced by

$$
\frac{\partial \psi_{0}}{\partial \phi}=\frac{\sin \theta}{\omega_{S}} \frac{d \psi_{0}}{d r}
$$

The linearized Vlasov equation therefore is,

$$
j \omega \psi_{p} e^{j \omega t}-\omega_{S} \frac{\partial \psi_{p}}{\partial \theta} e^{j \omega t}+\left(\phi+\omega_{s}^{2} \phi\right) \frac{\sin \theta}{\omega_{S}} \frac{d \psi_{0}}{d r}=0
$$

In the time domain, the coherent electromagnetic force represented by $\phi+\omega_{S}^{2} \phi$ can be written as,

$$
\phi+\omega_{S}^{2} \phi=-\frac{\omega_{S}^{2}}{V \cos \phi_{S}} V_{p}(\phi)
$$

where $V_{p}(\phi)$ represents the voltage induced by the perturbation term $\psi_{p}(r, \theta) e^{j \omega t}$ in (2-5). To determine $V_{p}(\phi)$, we define the line density $\lambda(\phi)$ as the projection of $\psi_{p}(r, \theta)$ on the $\phi$. 
axis,

$$
\lambda(\phi)=\int_{-\infty}^{\infty} \psi_{p}\left(\phi, \phi ; \omega_{S}\right) d \phi / \omega_{S}
$$

The line density can be Fourier expanded as.

$$
\lambda(\phi)=\frac{1}{2 \pi} \sum_{p=-\infty}^{\infty} \Lambda(p) e^{j p \phi}
$$

where the spectrum is,

$$
I(p)=\int_{-\infty}^{\infty} \lambda(\phi) e^{-j p \phi} d \phi
$$

Note that in the transform the variable is the phase deviation $\phi$, rather than the conventional time $t$. Using (2-10), we obtain,

$$
V_{p}(\phi)=-I_{0} e^{j+t} \sum_{p=-\infty}^{\infty} Z(p) \Lambda(p) e^{j p \phi}
$$

where $I_{0}$ is the beam average current and $Z(p)$ is the impedance corresponding to the spectrum $\Lambda(p)$. Substituting (2-12) into (2-8), we get,

$$
\ddot{\phi}+\omega_{S}^{2} \phi=-\frac{\omega_{S}^{2} I_{0}}{V \cos \phi_{S}} e^{j \omega t} \sum_{p=-\infty}^{\infty} Z(p) \Lambda(p) e^{j p \phi}
$$

We emphasize that $V_{p}(\phi)$ is the voltage generated by the line density $\lambda(\phi)$ in (2-9). which applies only to the particles with the phase position $\phi$

The perturbation distribution can be written,

$$
\psi_{p}(r, \theta)=\sum_{m^{\prime}=-\infty}^{\infty} R^{\left(m^{\prime}\right)}(r) e^{j m^{\prime} \theta}
$$

where $R^{\left(m^{\prime}\right)}(r)$ is the radial function with the $m^{\prime}$ th azimuthal mode. Substituting (2-13). (2-14) in to (2-7), and leaving off $e^{j \omega t}$, we get,

$$
j \sum_{m^{\prime}=-\infty}^{\infty}\left(\omega-m^{\prime} \omega_{S}\right) R^{\left(m^{\prime}\right)}(r) e^{j m^{\prime} \theta}=\frac{\omega_{S} I_{0}}{l \cos \phi_{S}} \sin \theta \frac{d \psi_{0}}{d r} \sum_{p=-\infty}^{\infty} Z(p) \Lambda(p) e^{j p \phi}
$$

Multiplying both sides of (2-15) by $e^{-j m \theta}$, and integrating over $\theta$ from 0 to $2 \pi$, we get,

$$
\left(\omega-m \omega_{S}\right) R^{(m)}(r)=j^{m+1} \frac{m \omega_{S} I_{0}}{V \cos \phi_{S}} \frac{d \psi_{0}}{d r} \frac{1}{r} \sum_{p=-\infty}^{\infty} \frac{Z(p)}{p} J_{m}(p r) \Lambda(p)
$$


where the identities

$$
\int_{0}^{2 \pi} e^{j(n-m) \theta} d \theta=2 \pi \delta_{n, m}
$$

and

$$
\int_{0}^{2 \pi} e^{-j(m \theta-p r \cos \theta)} \sin \theta d \theta=-\frac{2 \pi m}{p r} j^{m} J_{m}(p r)
$$

have been used.

The equation $(2-16)$ is the original Sacherer integral equation. In literature, the SIE is usually presented in the later form (2-23) or (3-1).

To determine the beam stability, the spectrum $\lambda(p)$ in $(2-16)$ is needed. Using (2-11), (2-9), (2-14), and the relation,

$$
d \phi d \phi / \omega_{S}=r d r d \theta
$$

we have,

$$
\begin{gathered}
\Lambda(p)=\int_{-\infty}^{\infty} \lambda(\phi) e^{-j p \phi} d \phi=\int_{-\infty}^{\infty}\left(\int_{-\infty}^{\infty} \psi_{p}\left(\phi, \phi / \omega_{S}\right) d \phi / \omega_{S}\right) e^{-j p \phi} d \phi \\
=\int_{0}^{\infty} r \sum_{m^{\prime}=-\infty}^{\infty} R^{\left(m^{\prime}\right)}(r) \int_{0}^{2 \pi} e^{j\left(m^{\prime} \theta-p r \cos 0\right)} d 0 d r=2 \pi \sum_{m^{\prime}=-\infty}^{\infty} j^{-m^{\prime}} \int_{0}^{\infty} R^{\left(m^{\prime}\right)}(r) J_{m^{\prime}}(p r) r d r
\end{gathered}
$$

If we define the Hankel spectrum of the radial function $R^{(m)}(r)$ as,

$$
\Lambda^{(m)}(p)=\int_{0}^{\infty} R^{(m)}(r) J_{m}(p r) r d r
$$

then the relation between the Fourier spectrum of the line density expanded on the phase deviation $\phi$ and the Hankel spectrum of the radial function is,

$$
\Lambda(p)=2 \pi \sum_{m^{\prime}--\infty}^{\infty} j^{-m^{\prime}} \Lambda^{\left(m^{\prime}\right)}(p)
$$

The Sacherer integral equation (2-16) can now be written as

$$
\begin{gathered}
\left(\omega-m \omega_{S}\right) R^{(m)}(r)=j^{m+1} m \omega_{s} \xi W(r) \sum_{p=-\infty}^{\infty} \frac{Z(p)}{p}-J_{m}(p r) \\
\times \sum_{m^{\prime}=-\infty}^{\infty} j^{-m^{\prime}} \int_{0}^{\infty} R^{\left(m^{\prime}\right)}\left(r^{\prime}\right) J_{m^{\prime}}\left(p r^{\prime}\right) r^{\prime} d r^{\prime}
\end{gathered}
$$

where we have used the weight function 


$$
W(r)=\frac{d \psi_{0}}{d r} \frac{1}{r}
$$

and also.

$$
\xi=\frac{2 \pi I_{0}}{V \cos \phi_{S}}
$$

\section{Solution Using Orthogonal Polynomials}

\subsection{Eigenvalue Problem}

If the azimuthal modes are not coupled, then only the force generated by the radial function $R^{(m)}(r)$ is responsible to the coherent motion $\omega$ in (2-23), the original Sacherer in tegral equation (2-16) is simplified as,

$$
\left(\omega-m \omega_{S}\right) R^{(m)}(r)=j m \omega_{S} \xi W(r) \sum_{p=-\infty}^{\infty} \frac{Z(p)}{p} J_{m}(p r) \int_{0}^{\infty} R^{(m)}\left(r^{\prime}\right) J_{m}\left(p r^{\prime}\right) r^{\prime} d r^{\prime}
$$

Thus, the SIE can be transformed to an eigenvalue problem, which is eligible to solve separately for two unknown variables, i.e., the coherent frequency $\omega$ and the radial function $R^{(m)}(r)$

For the weight function $W(r)$, a set of normalized orthogonal polynomials $f_{k}(r)$ can always be found such that,

$$
\int_{0}^{\infty} W(r) f_{k}(r) f_{l}(r) r d r=\delta_{k, l}
$$

Using the orthogonal polynomial, the radial function can be written as,

$$
R^{(m)}(r)=W(r) \sum_{k^{\prime}=0}^{\infty} \alpha_{k^{\prime}}^{(m)} f_{k^{\prime}}(r)
$$

Define the Hankel spectrum for the orthogonal polynomial,

$$
\Lambda_{k}^{(m)}(p)=\int_{0}^{\infty} W(r) f_{k}(r) J_{m}(p r) r d r
$$

Then we have

$$
\Lambda^{(m)}(p)=\sum_{k^{\prime}=0}^{\infty} \alpha_{k^{\prime}}^{(m)} \Lambda_{k^{\prime}}^{(m)}(p)
$$


The Bessel function $J_{m}(p r)$ can be cxpanded by the orthogcnal polynomials and the associated Hankel spectra.

$$
J_{m}(p r)=\sum_{k^{\prime}=0}^{\infty} \Lambda_{k^{\prime}}^{(m)}(p) f_{k^{\prime}}(r)
$$

Substituting (3-6) into (3-4), using (3-2), the expansion can be proved. It is interesting to notice that by this expansion the two variables $p$ and $r$ in the Bessel function are separated.

Substituting (3-3) and (3-6) into (3-1), we have,

$$
\begin{gathered}
\left(\omega-m \omega_{S}\right) W(r) \sum_{k^{\prime}=0}^{\infty} \alpha_{k^{\prime}}{ }^{(m)} f_{k^{\prime}}(r)=j m \omega_{S} \xi W(r) \sum_{p=-\infty}^{\infty} \frac{Z(p)}{p} \sum_{k^{\prime}=0}^{\infty} \Lambda_{k^{\prime}}{ }^{(m)}(p) f_{k^{\prime}}(r) \\
\times \int_{0}^{\infty} W\left(r^{\prime}\right) \sum_{k^{\prime}=0}^{\infty} \alpha_{k^{\prime}}(m) f_{k^{\prime}}\left(r^{\prime}\right) \sum_{l^{\prime}=0}^{\infty} \Lambda_{l^{\prime}}{ }^{(m)}(p) f_{l^{\prime}}\left(r^{\prime}\right) r^{\prime} d r^{\prime}
\end{gathered}
$$

Using (3-2), the equation becomes,

$$
\begin{gathered}
\left(\omega-m \omega_{S}\right) W(r) \sum_{k^{\prime}=0}^{\infty} \alpha_{k^{\prime}}{ }^{(m)} f_{k^{\prime}}(r)=j m \omega_{S} \xi W(r) \sum_{p=-\infty}^{\infty} \frac{Z(p)}{p} \\
\times \sum_{k^{\prime} l^{\prime}=0}^{\infty} \Lambda_{k^{\prime}}{ }^{(m)}(p) \Lambda_{l^{\prime}}{ }^{(m)}(p) f_{k^{\prime}}(r) \alpha_{l^{\prime}}{ }^{(m)}
\end{gathered}
$$

Define the matrix

$$
\mathbf{M}=\left|M_{k, l}{ }^{(m)}\right|=\left[\begin{array}{ccc}
\sum_{p=-\infty}^{\infty} \frac{Z(p)}{p} \Lambda_{1}{ }^{(m)}(p) \Lambda_{1}{ }^{(m)}(p) & \cdots & \sum_{p=-\infty}^{\infty} \frac{Z(p)}{p} \Lambda_{1}{ }^{(m)}(p) \Lambda_{\bar{l}}{ }^{(m)}(p) \\
\sum_{p=-\infty}^{\infty} \frac{Z(p)}{p} \Lambda_{\bar{k}}{ }^{(m)}(p) \Lambda_{1}{ }^{(m)}(p) & \cdots & \sum_{p=-\infty}^{\infty} \frac{Z(p)}{p} \Lambda_{\bar{k}}{ }^{(m)}(p) \Lambda_{\bar{l}}{ }^{(m)}(p)
\end{array}\right]
$$

i.e. we have

$$
M_{k, l}^{(m)}=\sum_{p=-\infty}^{\infty} \frac{Z(p)}{p} \Lambda_{k}^{(m)}(p) \Lambda_{l}^{(m)}(p)
$$

Multiplying (3-8) by $f_{l}(r) r$ for $l=1, \ldots, \bar{k}$, integrating over $r$, and using (3-9), we finaliy have the following equation, where $l$ is truncated at $\bar{k}$,

$$
\left(\omega-m \omega_{S}\right)\left[\begin{array}{c}
\alpha_{1}^{(m)} \\
\cdot \\
\cdot \\
\alpha_{\bar{k}}^{(m)}
\end{array}\right]=j m \omega_{S} \xi \mathbf{M}\left[\begin{array}{c}
\alpha_{1}{ }^{(m)} \\
\cdot \\
\alpha_{\bar{k}}^{(m)}
\end{array}\right]
$$


which is an eigenvalue problem. The eigenvalues can be solved through the equation

$$
\left|\lambda I-j m \omega_{s} \xi \mathbf{M}\right|=0
$$

where $I$ is identity matrix, and

$$
\lambda=\nu-m \omega_{S}
$$

We note that the eigenvector

$$
\alpha^{(m)}=\left[\begin{array}{c}
\alpha_{1}^{(m)} \\
\cdot \\
\alpha_{\bar{k}}^{(m)}
\end{array}\right]
$$

is not presented in (3-12) and therefore it will not directly affect the solution of the coherent frequency.

\subsection{Radial Modes}

Using the expansion of the radial function $R^{(m)}(r)$ by the orthogonal functions, as shown in (3-3), the Sacherer integral equation (3-1) has been transformed to a linear system (3-11) with an order $\bar{k}$. We have assumed that the higher order modes are not important with respect to the system response.

Define

$$
\mathbf{Z}=\operatorname{diag}\left\{\frac{Z(p)}{p}\right\}=\left[\begin{array}{cccc}
\frac{Z(-\bar{p})}{-\bar{p}} & 0 & \cdots & 0 \\
0 & \frac{Z(-\bar{p}+1)}{-\bar{p}+1} & \cdot & 0 \\
\cdot & \cdot & \cdot & \cdot \\
0 & 0 & \cdots & \frac{Z(\bar{p})}{\bar{p}}
\end{array}\right]
$$

and

$$
\Lambda=\left[\begin{array}{ccc}
\Lambda_{1}{ }^{(m)}(-\bar{p}) & \cdots & \Lambda_{1}{ }^{(m)}(\bar{p}) \\
\Lambda_{\bar{k}}^{(m)}(-\bar{p}) & \cdots & \Lambda_{\bar{k}}^{(m)}(\bar{p})
\end{array}\right]
$$


where we have truncated $p$ by $\pm \bar{p}$, and il is assumed that $2 \bar{p}+1>\bar{k}$. Now we have.

$$
\mathbf{M}=\Lambda \mathrm{Z} \Lambda^{T}
$$

where $\Lambda^{T}$ is the transpose of the matrix $\Lambda$. Under the condition of the inductive impedance. $Z(p) / p$ will be a constant, and therefore the matrix $Z$ is an identity matrix by a normalization. The rows in $\boldsymbol{\Lambda}$ represents a base of the system matrix $\mathbf{M}$. In general, the matrix $\mathbf{M}$ is nonsingular for almost all impedance, and therefore all eigenvalues will be nonzero.

Consider the system represented by (3-11). A simplest case is that the matrix $\mathbf{M}$ has $\bar{k}$ distinct eigenvalues, which guarantees the existence of a nonsingular matrix $T$ such that the following similarity transformation can be used for a matrix diagonalization as,

$$
\mathbf{M}_{d}=\mathrm{T}^{-1} \mathrm{M}_{\mathrm{l}} \mathrm{T}
$$

where for convenience we write,

$$
\mathbf{M}_{1}=j m \omega_{S} \xi \mathbf{M}
$$

and $\mathbf{M}_{d}$ is a diagonal matrix. The elements on the diagonal of $\mathbf{M}_{d}$ are the eigenvalues $\lambda_{(i)}, i=1, \ldots, \bar{k}$, which are all distinct as assumed. It is noticed that the eigenvectors are the columns of $T$. The equation (3-18) can be written as,

$$
\mathbf{M}_{1} \mathbf{T}=\mathrm{TM}_{d}=\mathbf{T} \operatorname{diag}\left\{\lambda_{(i)}\right\}
$$

which shows clearly that

$$
\mathrm{M}_{1} \mathrm{t}_{(i)}=\lambda_{(i)} \mathbf{t}_{(i)}
$$

where $t_{(i)}$ is the $i$ th column of $T$. The equation (3-21) indicates that $t_{(i)}$ is the eigenvector associated with the $i$ th eigenvalue $\lambda_{(i)}$.

In summary, if only the eigenvalues $\lambda_{(i)}$ are concerned, they can be found by using (312). Meanwhile using the similarity transformation (3-18), both the eigenvalues $\lambda_{(i)}$ and the corresponding eigenvectors $t_{(i)}$ can be found simultaneously.

It is interesting to notice that by comparing (3-21) with (3-11), we have

$$
t_{(i)}=\alpha_{(i)}^{(m)}
$$


where

$$
\alpha_{(i)}^{(m)}=\left[\begin{array}{c}
\alpha_{(i), 1}^{(m)} \\
\cdot \\
\cdot \\
\alpha_{(i), \bar{k}}^{(m)}
\end{array}\right]
$$

The corresponding radial function

$$
R_{(i)}^{(m)}(r)=W(r) \sum_{k^{\prime}=0}^{\infty} \alpha_{(i), k^{\prime}}^{(m)} f_{k^{\prime}}(r)
$$

is the $i$ th radial mode. Substituting the $i$ th eigenvalue $\lambda_{(i)}$ and the corresponding radial mode $R_{(i)}^{(m)}(r)$ into (3-1), the Sacherer integral equation is satisfied. This indicates that according to the SIE if the perturbation distribution is dominated by the $i$ th radial mode. i.e.

$$
\psi_{p}(r, 0)=R_{(i)}^{(m)}(r) e^{j m \theta}
$$

then only the coherent frequency shift $\lambda_{(i)}$ is excited, and other eigenvalues are virtually not presented.

To show the complication implied by the existence of $\bar{k}$ eigenvalues and also $\bar{k}$ associated eigenvectors, the following elaborations are needed. We rewrite the equation (3-11) as,

$$
\lambda \alpha=\mathrm{M}_{1} \alpha
$$

Taking Laplace transform, we have

$$
s \alpha(s)-\alpha_{0}=\mathrm{M}_{1} \alpha(s)
$$

where $\alpha_{0}$ is the initial condition of the vector $\alpha$ in time domain, i.e. $\alpha_{0}=\alpha\left(0_{-}\right)$. Since the orthogonal polynomials have been chosen, this can be interpreted as the initial perturbation distribution with respect to the radial modes, as shown in (2-14) and (3-3). Using partial fraction expansion, the evolution of the perturbation can be described as,

$$
\alpha(s)=\left(s \mathrm{I}-\mathrm{M}_{1}\right)^{-1} \alpha_{0}=\sum_{i=1}^{\bar{k}} \frac{\mathbf{t}_{(i)} \overline{\mathbf{t}}_{(i)}}{s-\lambda_{(i)}} \alpha_{0}
$$

where $\bar{t}_{(i)}$ is the $i$ th row of the matrix $\mathrm{T}^{-1}$, and the relation used in the second step can be 
easily shown by using (3-21).

By studying the equation (3-28), several results are presented as follows.

1. If the initial condition of the system can be written as

$$
\alpha_{0}=\kappa t_{(i)}
$$

where $\kappa$ is a constant, then only the $i$ th coherent frequency shift $\lambda_{(i)}$ is excited. To prove it. we write the time domain response of $(3-28)$ by using inverse Laplace transform as,

$$
\alpha(t)=\sum_{i=1}^{\bar{k}} \mathrm{t}_{(i)} \overline{\mathrm{t}}_{(i)} \alpha_{0} e^{j \lambda_{(i)} t}
$$

Using

$$
\overline{\mathbf{t}}_{\left(i, t_{(k)}\right.}=\delta_{i, k}
$$

which is self-evident considering their definitions, and substituting (3-29) into (3-30), the proof is completed.

Since $\alpha_{(i)}^{(m)}$ represents the $i$ th radial mode, as shown in (3-24), the condition (3-29) implies that only the $i$ th radial mode is presented in the perturbation. Therefore this result agrees with the previous observation concerning the fact that the $i$ th radial mode perturbation excites only the $i$ th coherent frequency shift.

2. For an arbitrary initial distribution of the radial modes, all coherent frequency shifts may be excited. The weighting of the $i$ th mode with respect to the system response is determined by

$$
w_{i}=\bar{t}_{(i)} \alpha_{0}
$$

This can be shown by using (3-3) and (3-30). The conclusion is that even though all the coherent frequency shifts can be found in solving the eigenvalues of the system matrix $M_{1}$, the information is not enough to determine the response of the system. The initial radial modes distribution has also to be considered in judging the importance of each mode, and therefore to determine the total response shown in (3-30).

3. If the distinctiveness of the eigenvalues is not guaranteed, the basic results still can go 
through for most cases. For instance, if two eigenvalues are the same as $\lambda_{(i)}$, instead of one with $\lambda_{(i)}$ and another with $\lambda_{(k)}$, then the second mode will be $t e^{j \lambda_{(i)} t}$ instead of $e^{j \lambda_{(k)} t}$

\section{Examples for Several Distributions}

In this section, we present solution of the Sacherer integral equation for several particle distributions by using the orthogonal polynomial approach. For each distribution, the corresponding orthogonal polynomials are found $|0|$. The remaining calculation are performed numerically by using the equations shown in the last section. Some closed forms for the Hankel spectra can be found in [7]. The physical implication of the radial modes will be discussed. For convenience, these discussion will be placed in the section of the Gaussian distribution.

\subsection{Gaussian Distribution}

For a Gaussian distribution, the stationary phase space density is

$$
\psi_{0}(r)=\frac{2}{\pi \hat{r}^{2}} e^{-2 r^{2} / \hat{r}^{2}}
$$

where $\hat{r}$ is the effective half bunch length in phase space. The line density is also a Gaus$\operatorname{sian}$,

$$
\lambda_{0}(\phi)=\frac{\sqrt{2}}{\sqrt{\pi}} \frac{1}{\phi} e^{-2 \phi^{2} / \phi^{2}}
$$

where $\phi$ is the effective half bunch length in phase deviation, which is in fact equal to $\hat{r}$. For Gaussian distribution, we have

$$
\phi=2 \sigma
$$

where $\sigma$ is the standard deviation of Gaussian distribution.

The weight function can be calculated as

$$
W(r)=\frac{-8}{\pi \hat{r}^{4}} e^{-2 r^{2} / \hat{r}^{2}}
$$

First, we find a set of orthogonal polynomials according to the weight function. The 
$-12$.

Hankel spectrum will be calculated numerically by using (3-4), and then the matrix $M$ of (3-9) is obtained. Using the similarity transformation (3-18), the eigenvalues and the eigenvectors are found, then using (3-24) lhe radial modes are found. The importance of each eigenvalue will be determined by evaluating the initial condition by (3-32).

Consider the following equation

$$
\int_{0}^{\infty} L_{k}{ }^{(\alpha)}(x) L_{l}^{(\alpha)}(x) e^{-x} x^{\alpha} d x=h_{k}{ }^{(\alpha)} \delta_{k, l}
$$

where $L_{k}^{(a)}(x)$ is the generalized Laguerre polynomial,

$$
L_{k}{ }^{(a)}(x)=\sum_{i=1)}^{k}(-1)^{i}\left(\begin{array}{c}
\alpha+k \\
k-i
\end{array}\right) \frac{x^{i}}{i !}
$$

and with

$$
h_{k}^{(\alpha)}=\frac{\Gamma(\alpha+k+1)}{k !}=\frac{(\alpha+k) !}{k !}
$$

Letting $x=2 r^{2} / \hat{r}^{2}$ and $\alpha=m$, and matching the equation (3-2) with (4-5), we have the orthogonal polynomials in this case as,

$$
f_{k}(r)=\left(\frac{\sqrt{2} r}{\hat{r}}\right)^{m}\left(\frac{\pi \hat{r}^{2} k !}{2(m+k) !}\right)^{1 / 2} L_{k}{ }^{(m)}\left(\frac{2 r^{2}}{\hat{r}^{2}}\right)
$$

The first 5 orthogonal polynomials are plotted in Fig.1, where and later we assumed that $\hat{r}=1$.

Using (3-4), the corresponding Hankel spectra can be found, which are shown in Fig.2. and the matrix $\Lambda$ is formed. Assuming an unity inductive impedance, the system matrix $M$ is formed. Take the AGS parameter at injection, i.e. $\int_{S}=1.78 \mathrm{KHz}, I_{0}=3.36 \mathrm{~A}$ with $6 \times 10^{13}$ protons per ring, and total RF voltage of $V=400 K V$. Using (3-18), the eigenvalues and the corresponding eigenvectors can be found. The first 4 eigenvalues of $M_{1}$ are $-5.26,-1.70,-0.29$, and -0.02 , respectively. The real eigenvalues imply that there is no mechanism to induce the instability. In Fig.3, the ridial modes, calculated by (3-24), are shown.

The radial modes can be observed through the corresponding line densities, which are 
obtained by,

$$
\lambda_{(i)}^{(m)}(\phi)=\frac{j^{m}}{2 \pi} \sum_{p=-\infty}^{\infty} \Lambda_{(i)}^{(m)}(p) e^{j p \phi}=\frac{j^{m}}{2 \pi} \sum_{p=-\infty}^{\infty} \sum_{k^{\prime}=0}^{\infty} \alpha_{(i), k^{\prime}}^{(m)} \Lambda_{k^{\prime}}^{(m)}(p) e^{j p o}
$$

The real part of the line densities are shown in Fig.t, where we used a uniform scaling for the strength of the perturbation. For a half period of a synchrotron oscillation, 4 snapshots of the corresponding line density at the clunally divided time period are shown in Fig.5, with only the first radial mode.

The following observations and deliberations are worth mentioning.

1. The initial condition of the perturbation $\alpha_{0}$ determines the weighting of each eigenvalue. The way to find $\alpha_{0}$ is by observing the line density generated by the initial perturbations, which are shown in Figs. 6-10 for first five radial modes separately. Note that the perturbation radial modes are shown on both side of (3-1), therefore the scaling is rather arbitrary. If the initial line density with perturbation is identified to be close to the waveform shown in Fig. $\overline{7}$, which is due to the second radial mode, then the second eigenvalue will be dominant in the beam motion. An identification can also be made through the spectrum analysis. With the linearity of the integral equation, each mode will manifest itself with either the damping or growth rate, which is determined by the corresponding eigenvalue. Consider an unstable case. When the strength of a mode increases, the particle distribution in phase space will be changed. This change can be shown as a growth of the amplitude of the corresponding radial mode, as shown in Fig.3. Ideally, a growth of a radial mode is not coupled to other modes.

2. In Figs. 6-10, although the arbitrary scaling was mentioned, care has been taken to avoid the appearance of a negative part of the line density. In fact, when a mode continues to grow, inevitably an empty portion in phase space will be generated. Any further manifest by the mode creates negative line density in theory. In reality, this cannot happen. Instead, distortion will appear on the boundary of the empty zone. In Fig.11, we show line densities with a strong 5 th radial mode along with the stationary distribution, which can be com- 
pared with Fig.10, where the line density has no negative portion. In Fig.12, a line density evolution in the AGS is shown, for the case of the intensity of $5.5 \times 10^{12}$ protons per ring, after transition [8|. The low order radial modes are clearly presented, also the distortion on the both side may be explained by the argument of the strong high order radial modes.

3. For the impedance due to a narrow band cavity, the impedance matrix $\mathbf{Z}$ can be calculated. Taking the AGS upgraded cavity as an example, where the quality factor is $Q=20$, and the shunt resistance is $R=4.2 \mathrm{~K} \Omega$. In the injection, the cavity will be detuned by $58.2 \mathrm{KHz}$ up from the RF frequency $f_{R F}=4.2 \mathrm{MHz}$ to accommodate the beam loading, and therefore the cavity resonant frequency is $f_{R}=4.2582 \mathrm{MHz}$. The matrix $\mathrm{Z}$ can be calculated, for instance, for $p=-10,-9, \ldots, 10$. The transfer function of the cavity is,

$$
Z(s)=\frac{2 \sigma R s}{s^{2}+2 \sigma s+\omega_{R}^{2}}
$$

where $\sigma=\omega_{R} /(2 Q)$ is the cavity half bandwidth. Substituting the Laplace operator $s$ in (4-10) by $j\left(\omega_{S}+p \omega_{R F}\right)$, the components of $\mathbf{Z}$ are obtained. Using (3-17), we get the matrix M. The radial modes are shown in Fig.13, which can be compared with Fig.3. It is observed that the first radial modes for inductive impedance and a narrow band cavity are quite different. The first four eigenvalues are $33.8 j-1170,0.06 j+45.8,0.005 j+i$, and $0.0001 j+0.3$, respectively. The imaginary part of the cigenvalues are all positive, because the detuning angle is in a correct direction. If the cavity is detuned downward, the eigenvalues become $-33.9 j+1448,-0.04 j+40,-0.004 j+6.4$, and $-0.0001 j+0.3$, respectively. The imaginary part of the eigenvalues are all negative, and the system is unstable. In Figs. 1418, the snapshots for line densities with the initial perturbation of the first five radial modes, respectively, are shown for a half synchrotron oscillation period.

\subsection{Parabolic Distributions}

In this section, we consider three different distributions, which are roughly identified in this article as the parabolic type of distributions. 
1. Elliptic distribution (Parabolic line density)

The stationary phase space density is

$$
L_{0}^{\prime}(r)=\frac{3}{2 \pi \hat{r}^{2}}\left(1-\frac{r^{2}}{\hat{r}^{2}}\right)^{1 / 2}
$$

The line density is,

$$
\lambda_{0}(\phi)=\frac{3}{4 \phi}\left(1-\frac{\phi^{2}}{\phi^{2}}\right)
$$

The weight function can be calculated as

$$
W(r)=\frac{-3}{2 \pi \dot{r}^{4}}\left(1-\frac{r^{2}}{\dot{r}^{2}}\right)^{-1 / 2}
$$

Consider

$$
\int_{-1}^{1} P_{k}^{(\alpha, \beta)}(x) P_{l}^{(\alpha, \beta)}(x)(1-x)^{\alpha}(1+x)^{\beta} d x=h_{k}{ }^{(\alpha, \beta)} \delta_{k, l}, \quad \alpha, \beta>-1
$$

where $P_{k}{ }^{(\alpha, \beta)}(x)$ is the Jacobi polynomial.

$$
P_{k}^{(a, \beta)}(x)=2^{-k} \sum_{i=0}^{k}\left(\begin{array}{c}
\alpha+k \\
i
\end{array}\right)\left(\begin{array}{c}
\beta+k \\
k-i
\end{array}\right)(x-1)^{k-i}(x+1)^{i}
$$

and with

$$
h_{k}^{(\alpha, \beta)}=\frac{2^{\alpha+\beta+1}}{2 k+\alpha+3+1} \frac{\Gamma(\alpha+k+1) \Gamma(\beta+k+1)}{k !+\Gamma(\alpha+\beta+k+1)}
$$

Letting $x=1-2 r^{2} / \hat{r}^{2}$, then we get

$$
\int_{0}^{i} W(r) f_{k}(r) f_{l}(r) r d r=\int_{-1}^{1} \frac{3}{2 \pi \hat{r}^{4}} \frac{\hat{r}^{2}}{2 \sqrt{2}}(x+1)^{-1 / 2} f_{k}(x) f_{l}(x) d x
$$

Matching (4-17) with (4-14), we have $\beta=-1 / 2$, and also

$$
f_{k}(x)=\left(\frac{4 \sqrt{2} \pi \hat{r}^{2}}{3} \frac{(\alpha+2 k+1 / 2) k ! \Gamma(\alpha+k+1 / 2)}{2^{\alpha+1 / 2} \Gamma(\alpha+k+1) \Gamma(k+1 / 2)}\right)^{1 / 2}(1-x)^{\alpha / 2} P_{k}^{(\alpha,-1 / 2)}(x)
$$

Letting $\alpha=m$, we have,

$$
f_{k}(r)=\left(\frac{4 \pi(m+2 k+1 / 2) k ! \Gamma(m+k+1 / 2)}{3(m+k) ! \Gamma(k+1 / 2)}\right)^{1 / 2} \hat{r}\left(\frac{r}{\hat{r}}\right)^{m} P_{k}^{(m,-1 / 2)}\left(1-\frac{2 r^{2}}{\hat{r}^{2}}\right)
$$

In this and following examples, we use expansion of 7 orthogonal polynomials with $p= \pm 10$.The first 5 orthogonal polynomials are plotted in Fig.19. The radial modes with 
inductive impedance are shown in Fig.20, and the corresponding line densities are shown in Fig.21.

2. Parabolic distribution

The stationary phase space density is

$$
\psi_{0}(r)=\frac{2}{\pi \hat{r}^{2}}\left(1-\frac{r^{2}}{\hat{r}^{2}}\right)
$$

The line density is,

$$
\lambda_{0}(\phi)=\frac{8}{3 \pi \phi}\left(1-\frac{\phi^{2}}{\phi^{2}}\right)^{3 / 2}
$$

and the weight function is,

$$
W(r)=\frac{-1}{\pi \dot{r}^{\prime}}
$$

Letting $x=1-2 r^{2} / \hat{r}^{2}$, then we get

$$
\int_{0}^{\dot{r}} W(r) f_{k}(r) f_{l}(r) r d r=\int_{-1}^{1} \frac{1}{\pi \hat{r}^{2}} f_{k}(x) f_{l}(x) d x
$$

Matching (4-23) with (4-14), we have $\beta=0$. Letting $\alpha=m$, we have,

$$
f_{k}(r)=\left(\frac{\pi(m+2 k+1)}{2}\right)^{1 / 2} \hat{r}\left(\frac{r}{\hat{r}}\right)^{m} P_{k}^{(m, 0)}\left(1-\frac{2 r^{2}}{\hat{r}^{2}}\right)
$$

The orthogonal polynomials are plolled in Fig.22. The radial modes with inductive impedance are shown in Fig.23, and the corresponding line densities are shown in Fig.24.

3. Smoothed parabolic distribution

The stationary phase space density is

$$
\psi_{0}(r)=\frac{5}{2 \pi \hat{r}^{2}}\left(1-\frac{r^{2}}{\hat{r}^{2}}\right)^{3 / 2}
$$

The line density is,

$$
\lambda_{0}(\phi)=\frac{15}{16}\left(1-\frac{\phi^{2}}{\phi^{2}}\right)^{2}
$$

The weight function can be calculated as

$$
W(r)=\frac{-15}{2 \pi \hat{r}^{4}}\left(1-\frac{r^{2}}{\dot{r}^{2}}\right)^{1 / 2}
$$


We have

$$
\int_{0}^{\dot{r}} W(r) f_{k}(r) f_{l}(r) r d r=\int_{-1}^{1} \frac{15}{8 \sqrt{2} \pi r^{2}}(1+x)^{1 / 2} f_{k}(x) f_{l}(x) d x
$$

Letting $\alpha=m$ and $\beta=1 / 2$, we have

$$
f_{k}(r)=\left(\frac{4 \pi(m+2 k+3 / 2) k ! \Gamma(m+k+3 / 2)}{15 \Gamma(m+k+1) \Gamma(k+3 / 2)}\right)^{1 / 2} \hat{r}\left(\frac{r}{\hat{r}}\right)^{m} P_{k}^{(m, 1 / 2)}\left(1-\frac{2 r^{2}}{\hat{r}^{2}}\right)
$$

The orthogonal polynomials are plotted in Fig.25. The radial modes with inductive impedance are shown in Fig.26, and the corresponding line densities are shown in Fig.27.

\section{Solution Using Hankel Harmonic Samplings}

The Sacherer integral equation (3-1) can also be solved by Hankel harmonic sampling of the radial modes at $p$, which represents frequencies, in an eigenvalue problem. We rewrite the equation (3-1) as,

$$
\lambda R^{(m)}(r)=j m \omega_{S} \xi W(r) \sum_{p=-\infty}^{\infty} \frac{Z(p)}{p} J_{m}(p r) \Lambda^{(m)}(p)
$$

Multiplying both side by $J_{m}(q r) r$ and integrating over $r$, using (2-21), we get,

$$
\lambda \Lambda^{(m)}(q)=j m \omega_{s} \xi \sum_{p=-\infty}^{\infty} \frac{Z(p)}{p} \int_{0}^{\infty} W(r) J_{m}(p r) J_{m}(q r) r d r \cdot \Lambda^{(m)}(p)
$$

Running $q$ from $-\bar{p}$ to $\bar{p}$, then for $p$ from $-\bar{p}$ to $\bar{p}$, we have,

$$
\lambda\left[\begin{array}{c}
\Lambda^{(m)}(-\bar{p}) \\
\cdot \\
\Lambda^{(m)}(\bar{p})
\end{array}\right]=j m \omega_{S} \xi \mathrm{K}\left[\begin{array}{c}
\Lambda^{(m)}(-\bar{p}) \\
\cdot \\
\cdot \\
\Lambda^{(m)}(\bar{p})
\end{array}\right]=j m \omega_{S} \xi\left[\begin{array}{ccc}
K_{-\bar{p},-\bar{p}}^{(m)} & \cdots & K_{\bar{p},-\bar{p}}^{(m)} \\
\cdot & \cdot & \cdot \\
\cdot & \cdot & \cdot \\
K_{-\bar{p}, \bar{p}}^{(m)} & \cdots & K_{\bar{p}, \bar{p}}^{(m)}
\end{array}\right]\left[\begin{array}{c}
\Lambda^{(m)}(-\bar{p}) \\
\cdot \\
\cdot \\
\Lambda^{(m)}(\bar{p})
\end{array}\right]
$$

where

$$
K_{p, q}^{(m)}=\frac{Z(p)}{p} \int_{0}^{\infty} W(r) J_{m}(p r) J_{m}(q r) r d r
$$

The equation (5-3) is also an eigenvalue problem.

To find the relation between the two approaches, we have the following equation according to (3-5), 


$$
\left[\begin{array}{c}
\Lambda^{(m)}(-\bar{p}) \\
\Lambda^{(m)}(\bar{p})
\end{array}\right]=\Lambda^{T}\left[\begin{array}{c}
\alpha_{1}^{(m)} \\
\cdot \\
\alpha_{\bar{k}}^{(m)}
\end{array}\right]
$$

where the matrix $\Lambda^{T}$ is defined in (3-16). Using the equation (3-6), we may write

$$
\begin{gathered}
K_{p, q}^{(m)}=\frac{Z(p)}{p} \int_{0}^{\infty} W\left(r^{\prime}\right) \sum_{k^{\prime}=0}^{\infty} \Lambda_{k^{\prime}}{ }^{(m)}(p) f_{k^{\prime}}\left(r^{\prime}\right) \sum_{l^{\prime}=0}^{\infty} \Lambda_{l^{\prime}}{ }^{(m)}(q) f_{l^{\prime}}\left(r^{\prime}\right) r^{\prime} d r^{\prime} \\
=\frac{Z(p)}{p} \sum_{k^{\prime}=0}^{\infty} \Lambda_{k^{\prime}}{ }^{(m)}(p) \Lambda_{k^{\prime}}{ }^{(m)}(q)
\end{gathered}
$$

Thus, we have,

$$
\mathbf{K}=\left|K_{p, q}^{(m)}\right|=\left[\begin{array}{ccc}
\frac{Z(-\bar{p})}{-\bar{p}} \sum_{k^{\prime}=0}^{\infty} \Lambda_{k^{\prime}}{ }^{(m)}(-\bar{p}) \Lambda_{k^{\prime}}{ }^{(m)}(-\bar{p}) & \cdots & \frac{Z(\bar{p})}{\bar{p}} \sum_{k^{\prime}=0}^{\infty} \Lambda_{k^{\prime}}{ }^{(m)}(-\bar{p}) \Lambda_{k^{\prime}}{ }^{(m)}(\bar{p}) \\
\frac{Z(-\bar{p})}{-\bar{p}} \sum_{k^{\prime}=0}^{\infty} \Lambda_{k^{\prime}}{ }^{(m)}(\bar{p}) \Lambda_{k^{\prime}}{ }^{(m)}(-\bar{p}) & \cdots & \frac{Z(\bar{p})}{\bar{p}} \sum_{k^{\prime}=0}^{\infty} \Lambda_{k^{\prime}}{ }^{(m)}(\bar{p}) \Lambda_{k^{\prime}}{ }^{(m)}(\bar{p})
\end{array}\right]
$$

which can be decomposed as, assuming that $k^{\prime}$ is truncated at $\bar{k}$,

$$
\mathbf{K}=\Lambda^{T} \Lambda \mathbf{Z}
$$

Finally the equation (5-3) can be written as,

$$
\lambda \boldsymbol{\Lambda}^{T} \alpha^{(m)}=j m \omega_{S} \xi \mathbf{K} \boldsymbol{\Lambda}^{T} \alpha^{(m)}=j m \omega_{S} \xi \boldsymbol{\Lambda}^{T} \mathbf{\Lambda} \mathbf{Z} \Lambda^{T} \alpha^{(m)}=j m \omega_{S} \xi \Lambda^{T} \mathbf{M} \alpha^{(m)}
$$

By canceling $\Lambda^{T}$, which in almost all cases has a full column rank, the equation (5-9), and therefore (5-3), becomes (3-11), and the relation between the two approaches is presented.

In the following, we make several comments on the two approaches.

1. Consider the case of an inductive impedance. We note that the dimensions of the matrices $\mathbf{M}$ and $\mathbf{K}$ are $\bar{k} \times \bar{k}$ and $2 \bar{p}+1 \times 2 \bar{p}+1$, respectively. Consider the condition that $K_{p, q}^{(m)}$ in $(5-3)$ is represented by (5-6), and $k^{\prime}$ is truncated at $\bar{k}$. For a special case of $2 \bar{p}+1=\bar{k}$, the two approaches are equivalent. In the general case of $2 \bar{p}+1 \neq \bar{k}$, disregarding the trivial zero eigenvalues, the two matrices can be made similar, i.e. related by a similarity transformation, and therefore they have a same characteristic polynomial and a same 
set of eigenvalues. Even though the dimensions of lhe two matrices are different. by an inspection it can be lound that the computing load is the same, i.e. both need the Hankel spectra for orthogonal polynomials shown in (3-4).

2. Using the harmonic sampling approach, the matrix $\mathbf{K}$ can be calculated by the Bessel functions as shown in (5-4). The equation (3-6) shows that the Bessel function is equivalent to an expansion of the orthogonal polynomials with an infinite order. Meanwhile in the orthogonal polynomial approach, $k$ hiss to be truncated. If the convergence in the orthogonal polynomial expansion is guaranteed and it is fast, then the difference between the two approaches will not be important. Otherwise, the Bessel function calculation can be used to verify the accuracy of the orthogonal polynomial approach. In Fig.28, the Bessel function $J_{1}(p r)$ with $p=1,2,3,4,5$ are shown, compared with the dotted lines for the orthogonal polynomial approximation using (3-6), where the Gaussian distribution and up to 5 th order orthogonal polynomials are used. It can be observed that to let the approximation acceptable, in this case, for the harmonic number larger than 5 a higher order orthogonal polynomial expansion is needed.

3. In the orthogonal polynomial approach, sometimes the harmonic number can be extended to be very large by an analytical expression of the component in the matrix.$M$. Consider an inductive impedance, and assume that the perturbation line density of each orthogonal polynomial is known. We may write

$$
\begin{aligned}
M_{k, l}^{(m)}=\sum_{p=-\infty}^{\infty} \Lambda_{k}{ }^{(m)}(p) \Lambda_{l}{ }^{(m)}(p) & =\sum_{p=-\infty}^{\infty} \int_{-\infty}^{\infty} \lambda_{k}{ }^{(m)}(\phi) e^{-j p \phi} d \phi \int_{-\infty}^{\infty} \lambda_{l}{ }^{(m)}\left(\phi^{\prime}\right) e^{-j p \phi^{\prime}} d \phi^{\prime} \\
& =2 \pi \int_{-\infty}^{\infty} \lambda_{k}{ }^{(m)}(\phi) \lambda_{l}{ }^{(m)}(-\phi) d \phi
\end{aligned}
$$

The last step in (5-10) requires that the harmonic number $p$ be very large, therefore this expression includes very high harmonics. Whereas in the harmonic sampling approach, the number of the orthogonal polynomials is infinite, but the harmonic number is limited. This condition for using $(5-10)$ is crucial and very restricted, therefore the usage of this method is limited. 


\section{Acknowledgment}

Nie would like to thank E.C. Raka for helpful discussions.

\section{References}

[1! F. Sacherer, 'Bunch Lengthening and Vicrowave Instability, ' IEEE Trans. Vuclear Sience, Vol. 24, pp.1393-1395, 1977.

[2] A.W. Chao, 'Physics of Collective Beam Instabilities in High Energy Accelerators. John Wiley \& Sons. New York, 1993.

(3) J.L. Laclare, 'Bunched Beam Coherent Instabilities,' CERN 87-03, pp.264-326, 1987

(4) G. Besnier, Stabilité des Oscillations Longitudinales d'un Faisceau Groupe se Pro. pageant dans une Chambre a vide D'impedance Reactive, Nucl. Instru. Methods. Vol.164, pp.235, 1979.

[5] B. Zotter, 'Longitudinal Stability of Bunched Beams, I: Resonator Impedances,' CER.' SPS $/ 81-18,1981$.

(6) I. Gradshteyn and I. Ryzhik, 'Tables of Integrals, Series, and Products.' Academic Press, New York, 1980.

I: K. Satoh, Stability of Higher-order Longitudinal . Wodes in a Bunched Beam Withi:t Iode Coupling,' PEP Note 35T, 1981

8! E.C. Raka, Private Communication 


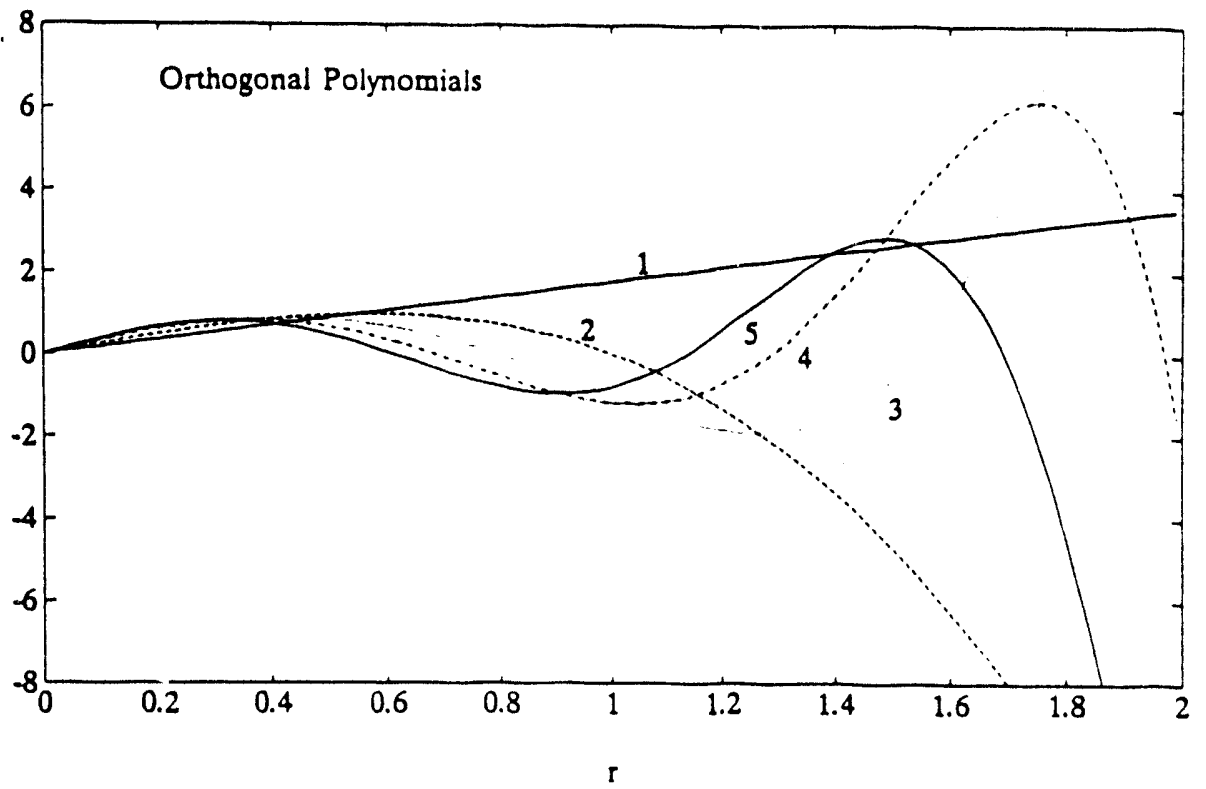

Fig.1. Orthogonal Polynomials for Gaussian Distribution.

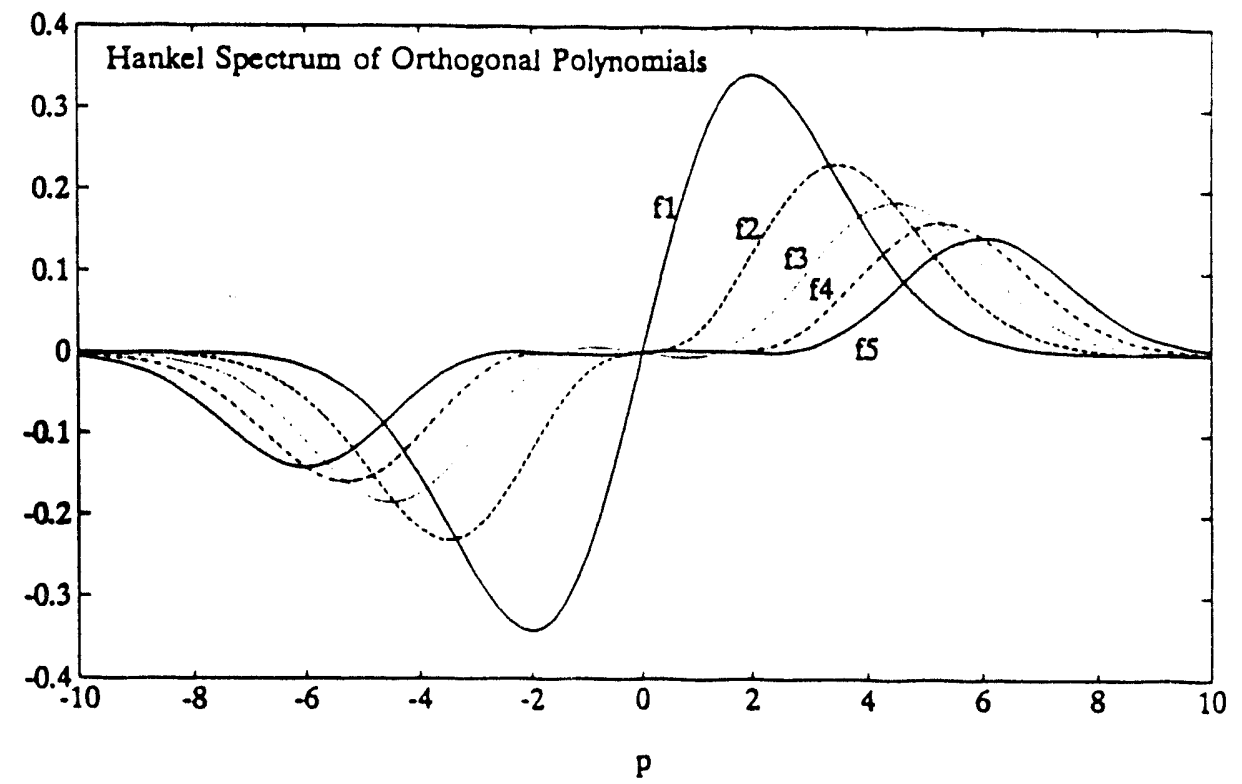

Fig.2. Hankel Spectra of Orthogonal Polynomials for Gaussian Distribution. 


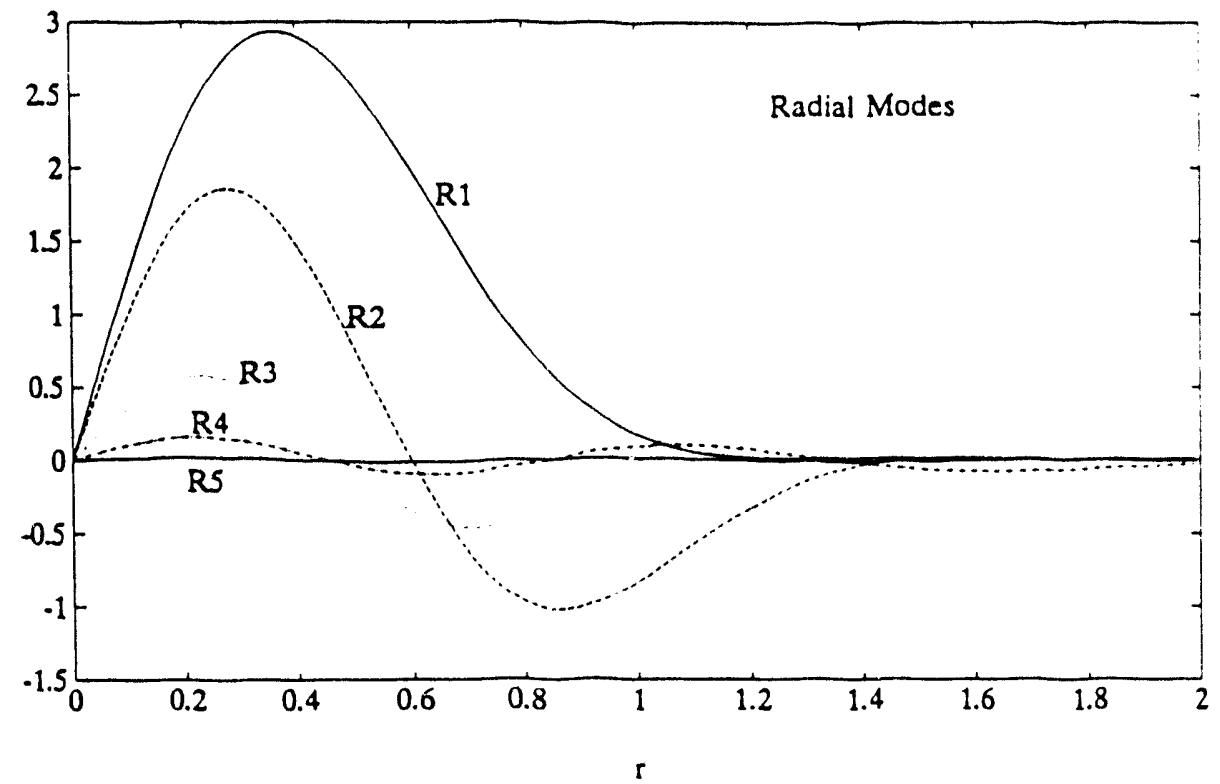

Fig.3. Radial Modes of Gaussian Distribution.

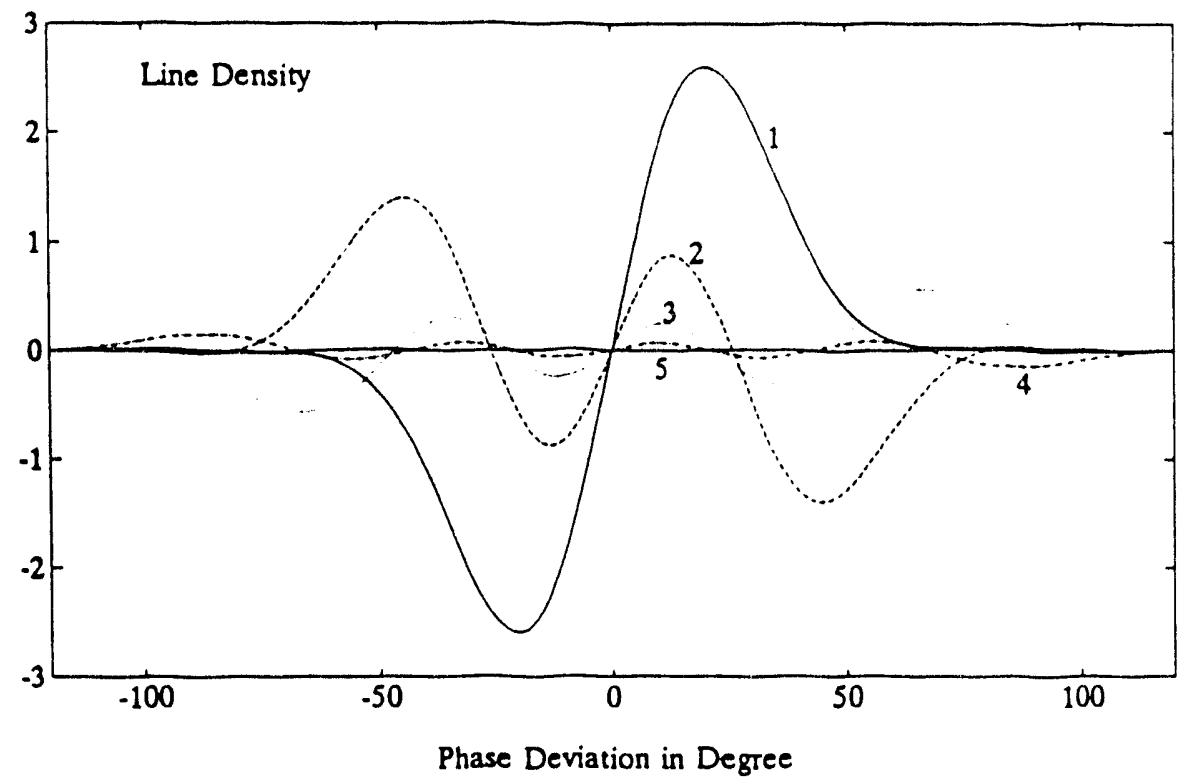

Fig.4. Line Density of Radial Modes for Gaussian Distribution. 


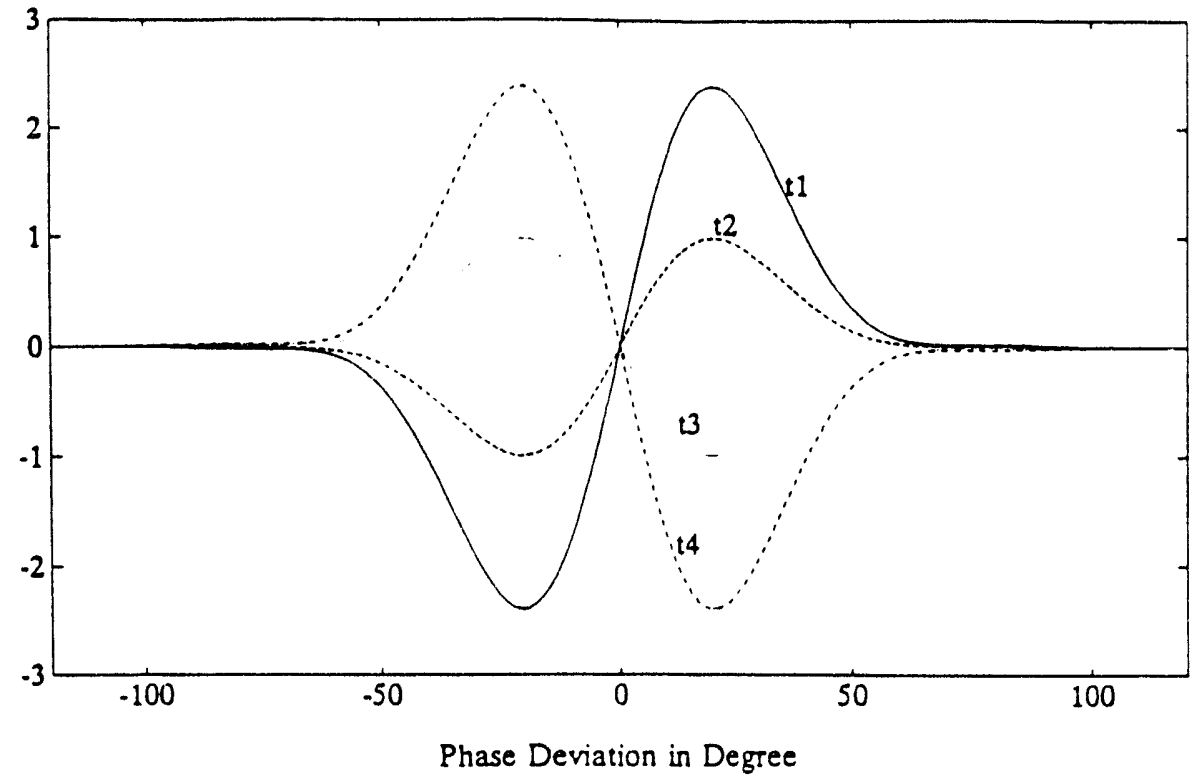

Fig.5. Snapshot of Line Density of 1st Radial Mode for Gaussian Distribution.

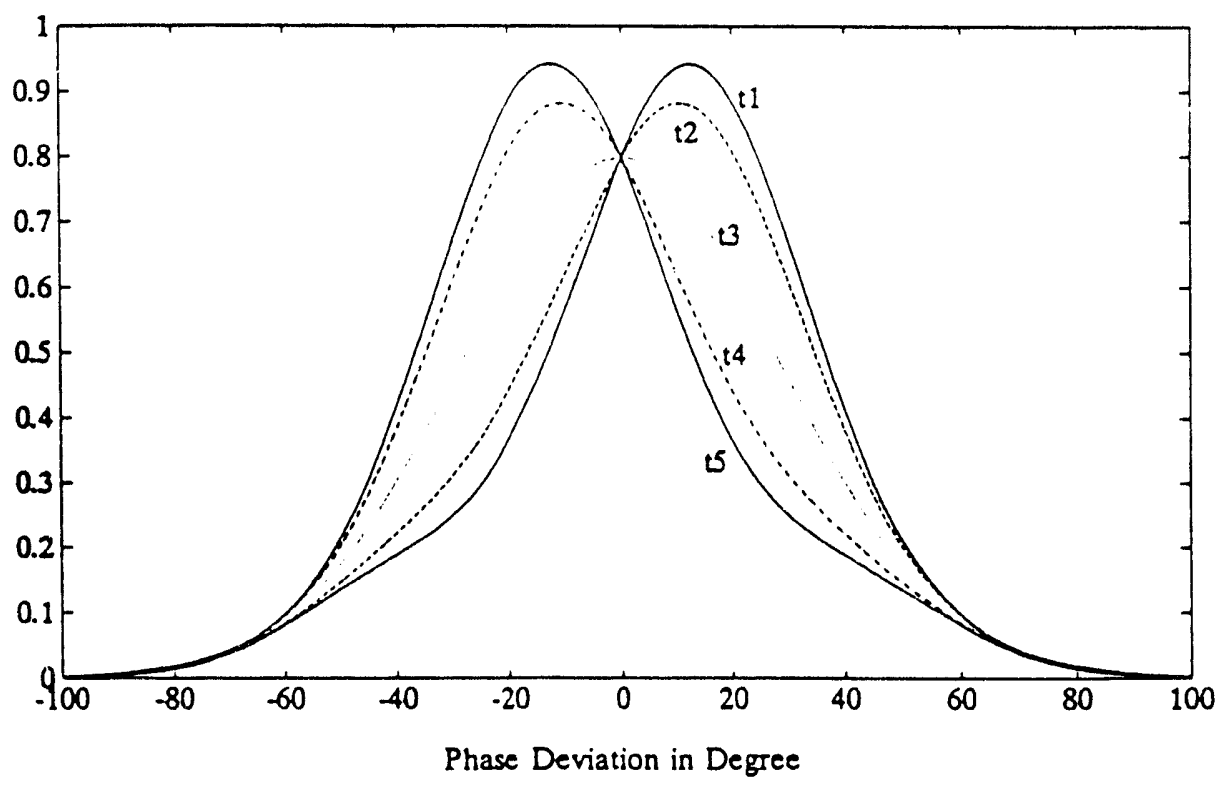

F:g.6. Line Density of Stationary Distribution and 1st Radial Mode, Gaussian. 


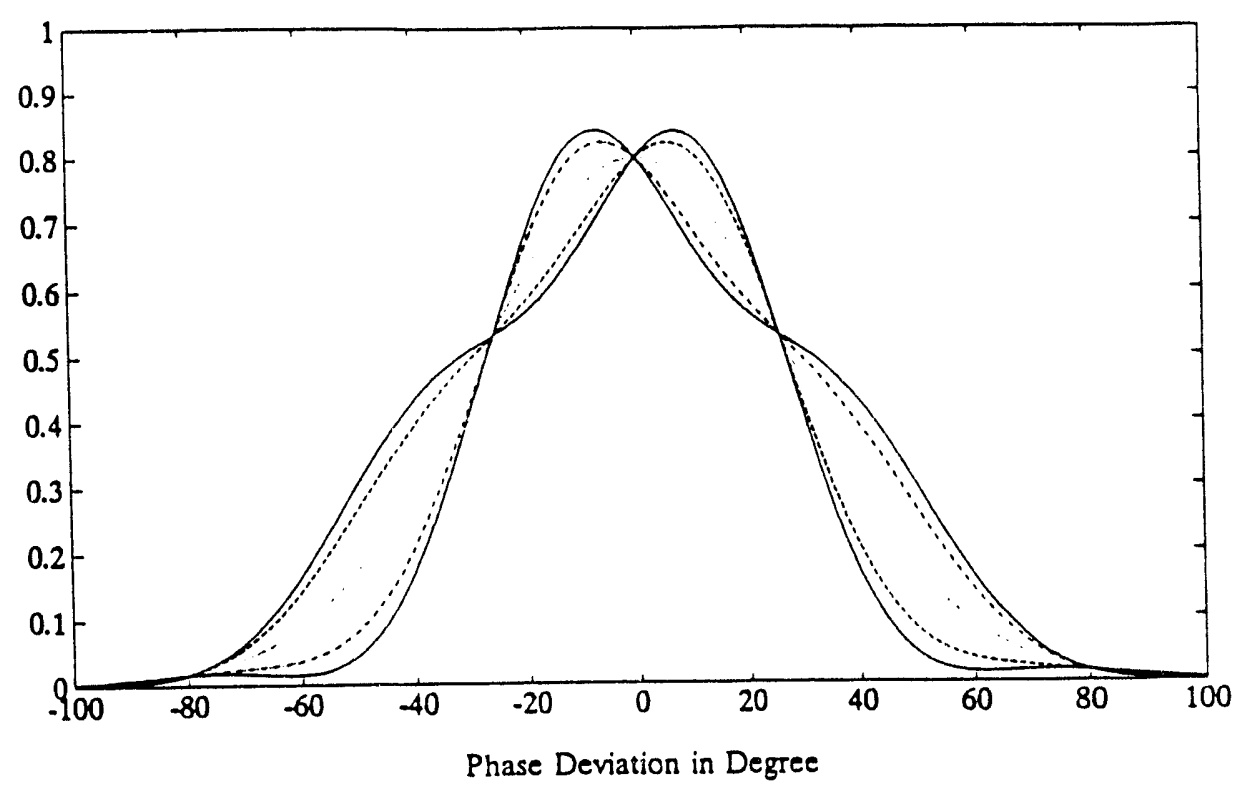

Fig.7. Line Density of Stationary Distribution and 2nd Radial Mode, Gaussian.

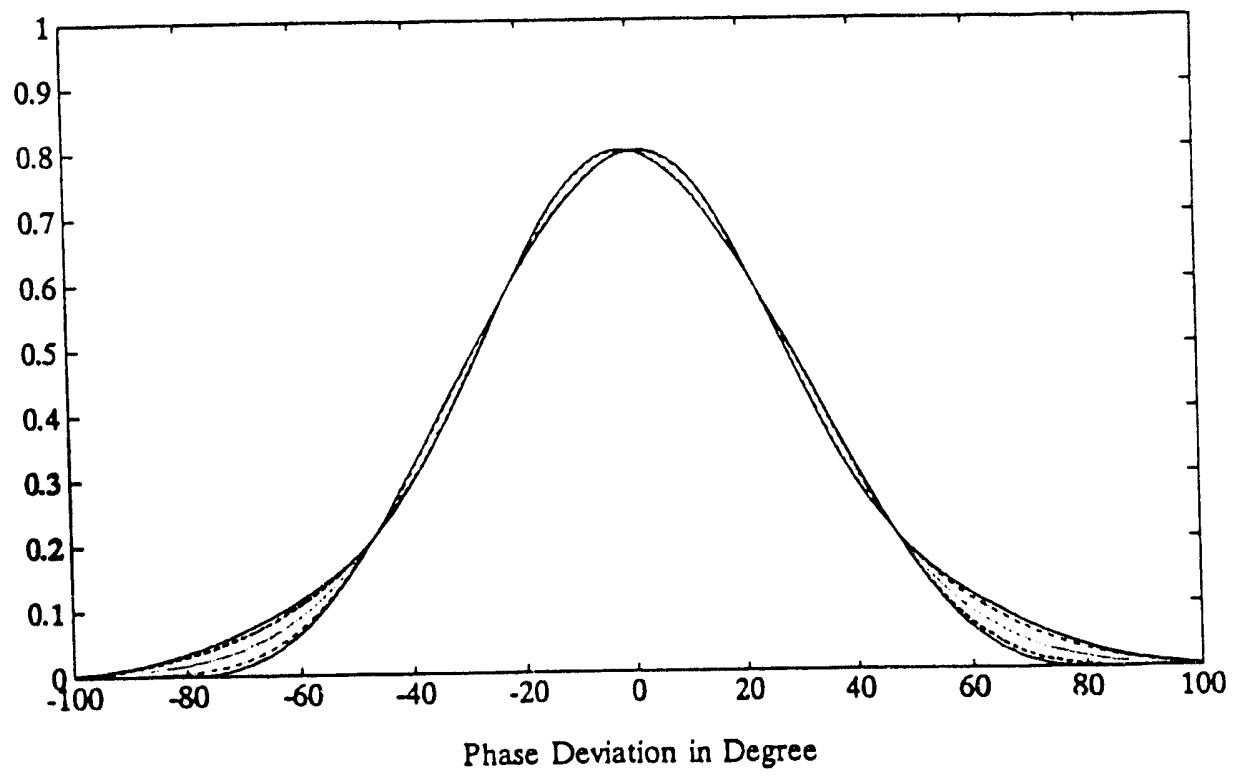

Fig.8. Line Density of Stationary Distribution and 3rd Radial Mode, Gaussian. 


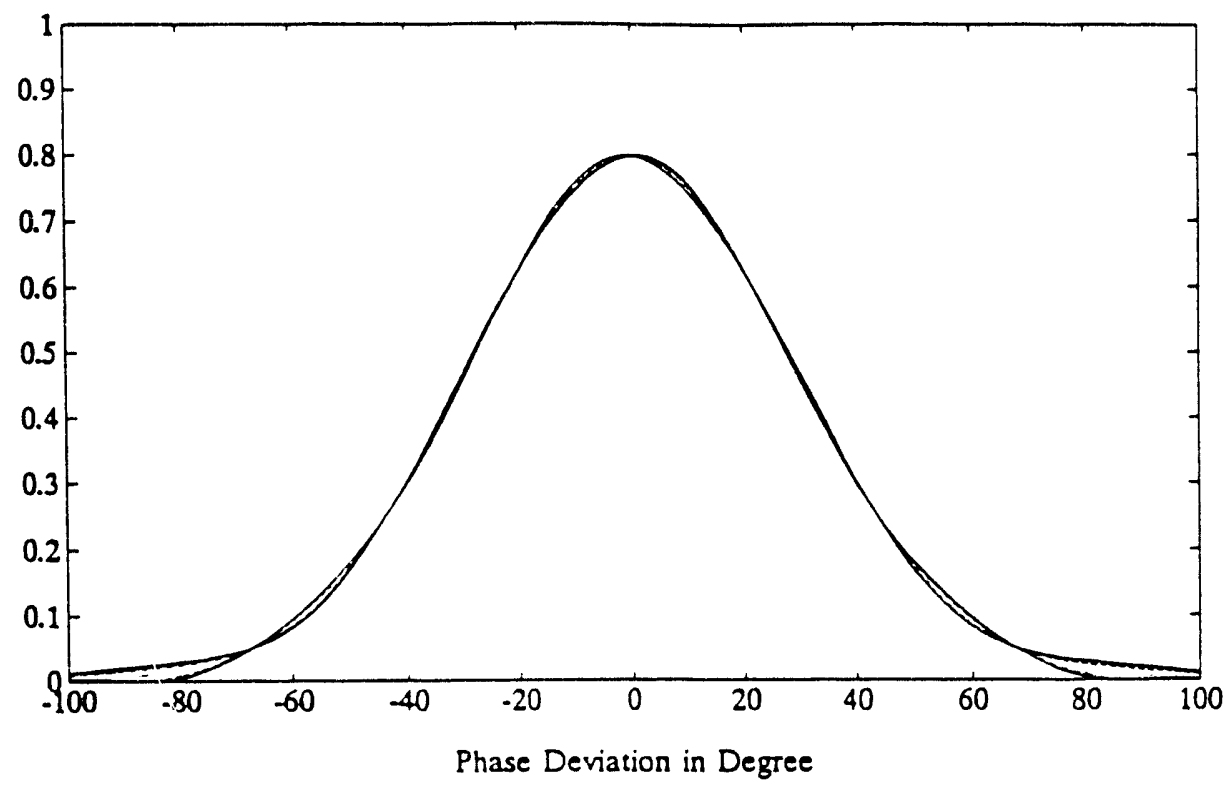

Fig.9. Line Density of Stationary Distribution and 4th Radial Mode, Gaussian.

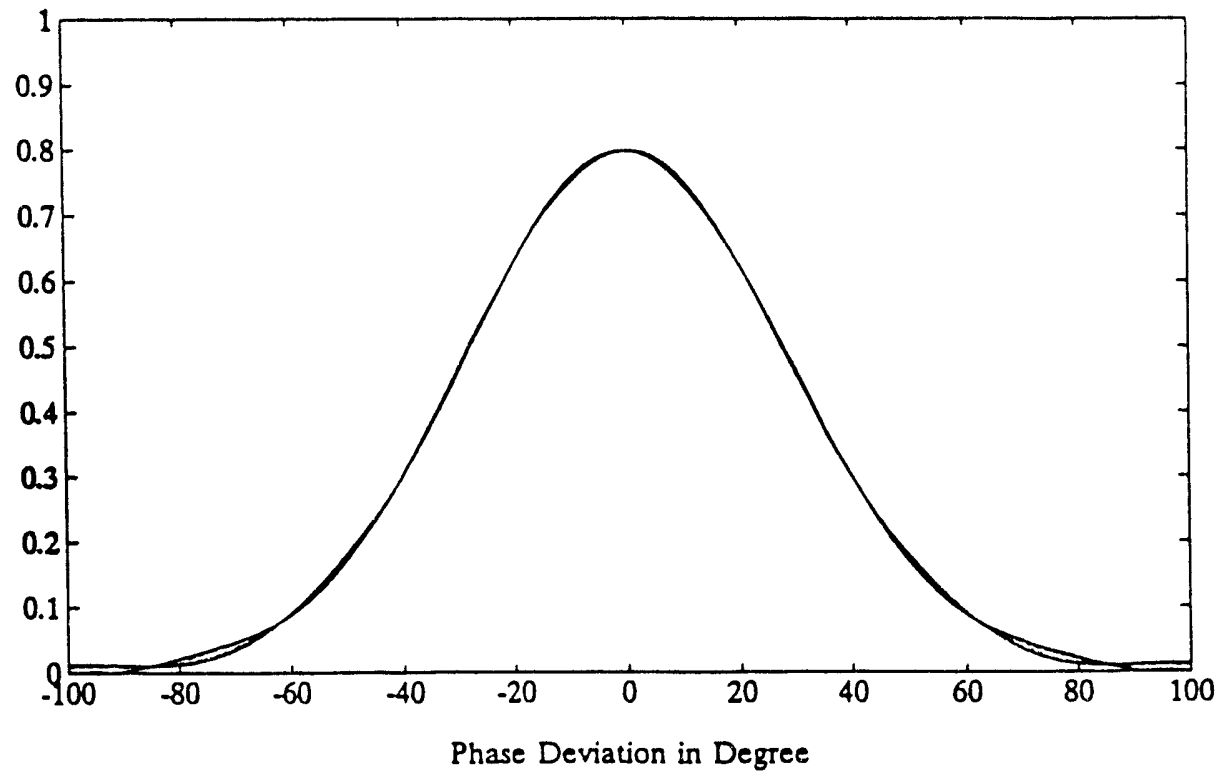

Fig.10. Line Density of Stationary Distribution and 5th Radial Mode, Gaussian. 


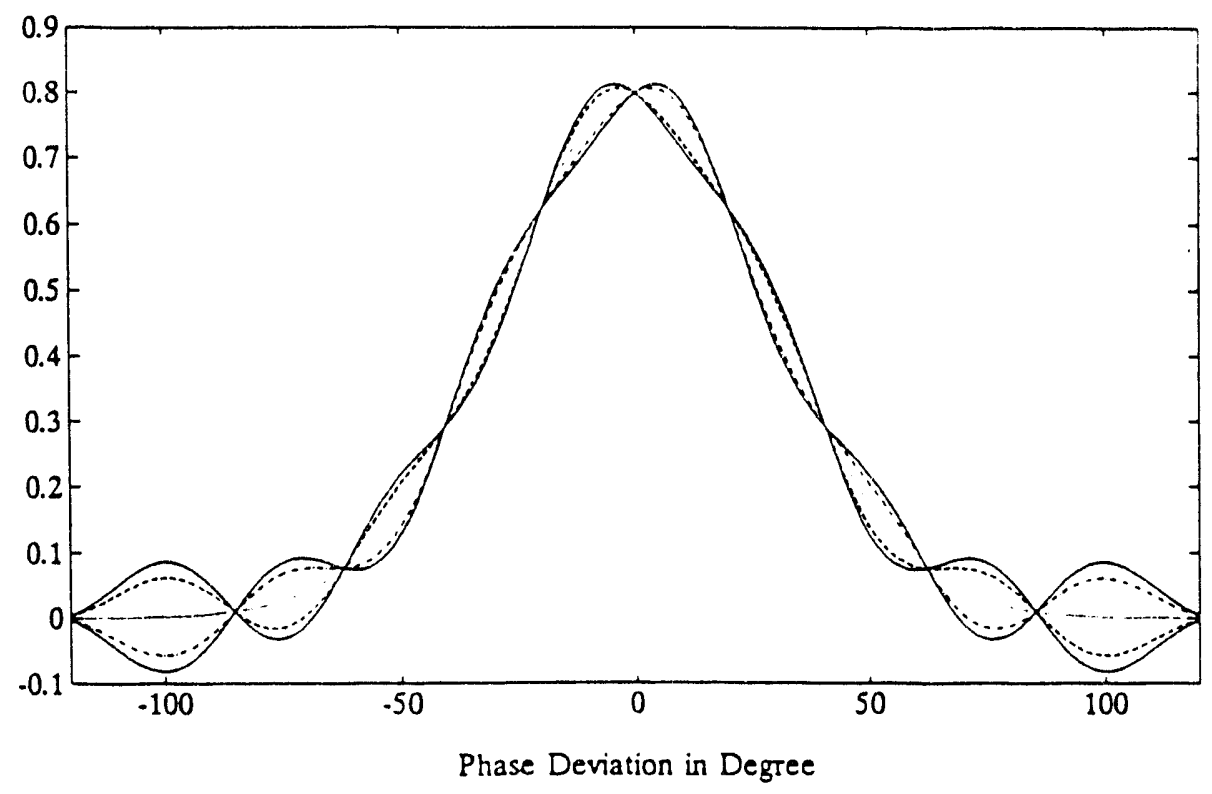

Fig.11. Line Density of Stationary Distribution and Strong 5th Radial Mode.

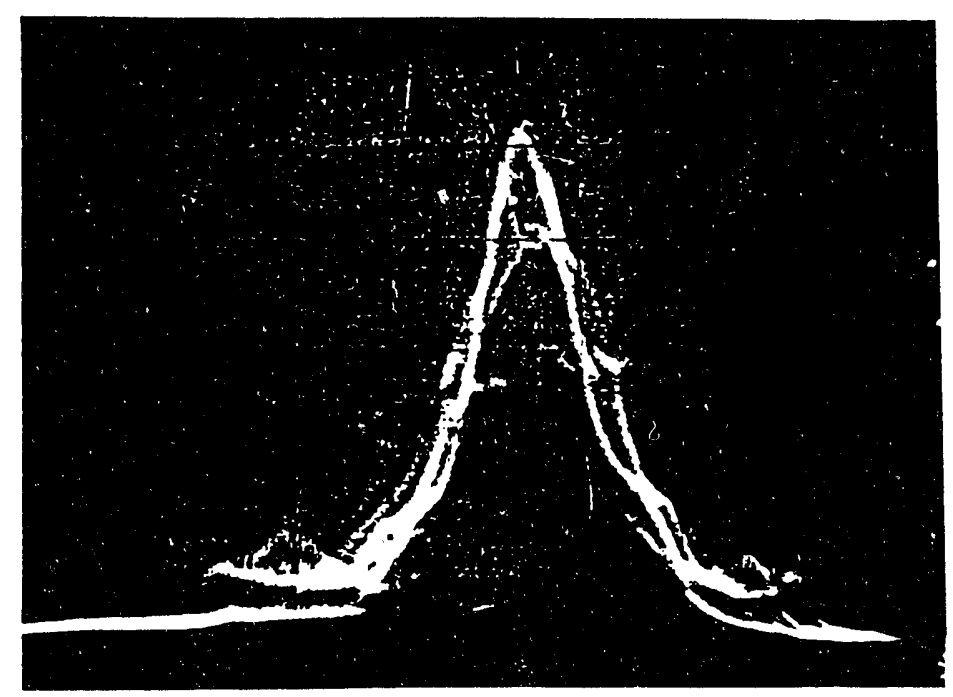

Fig.12. Evolution of Line Density at the AGS. 


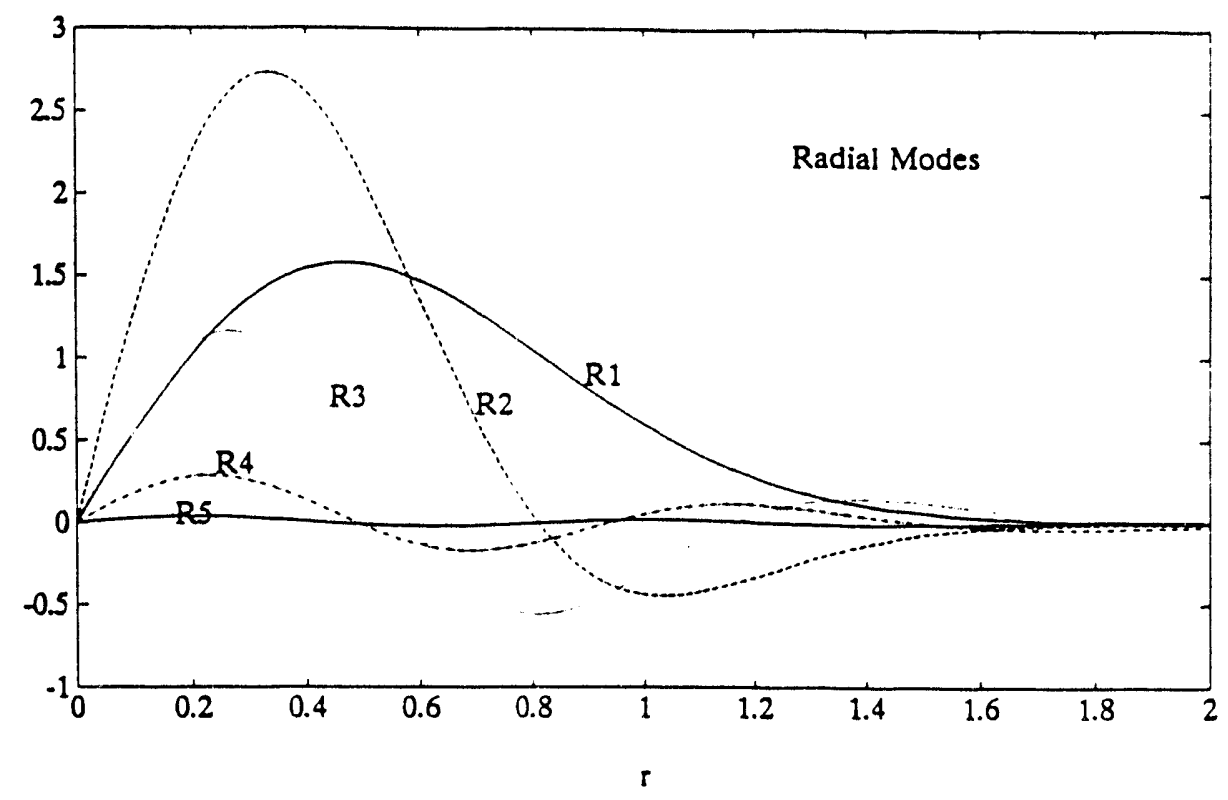

Fig.13. Radial Modes with a Narrow Band Cavity, Gaussian.

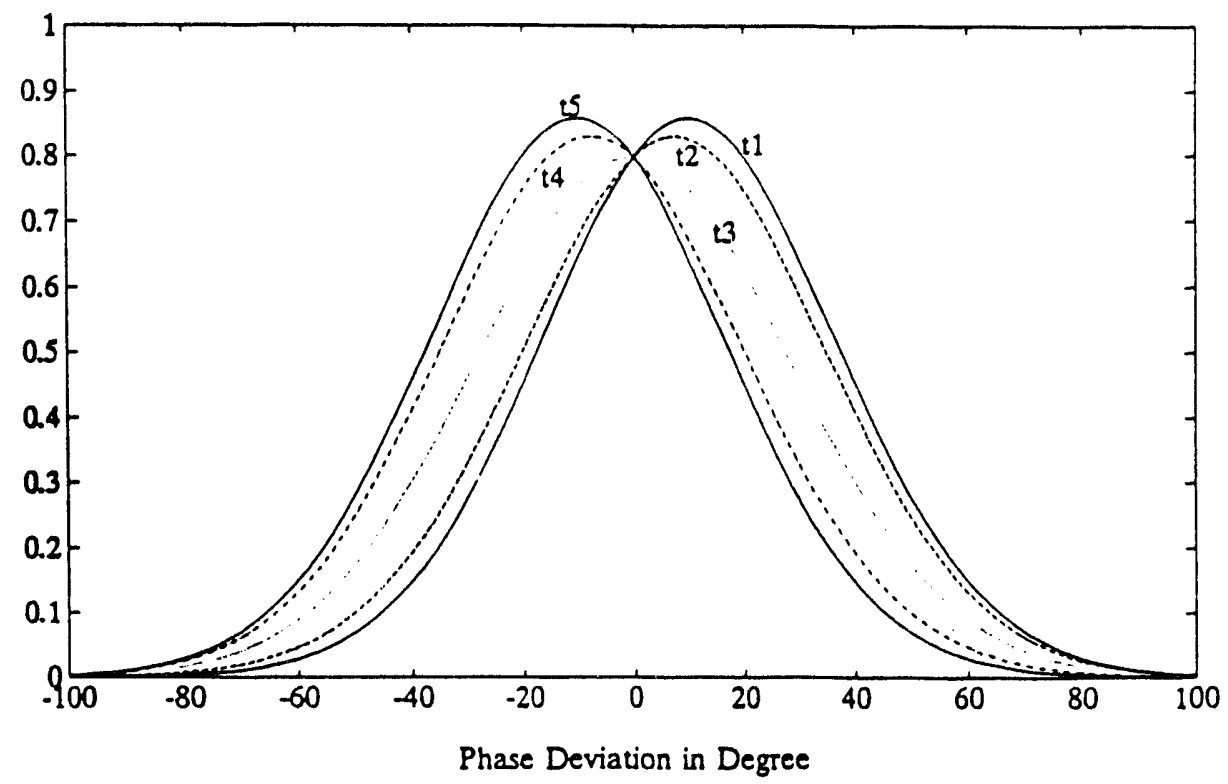

Fig.14. Line Density of Stationary Distribution and 1st Radial Mode. 


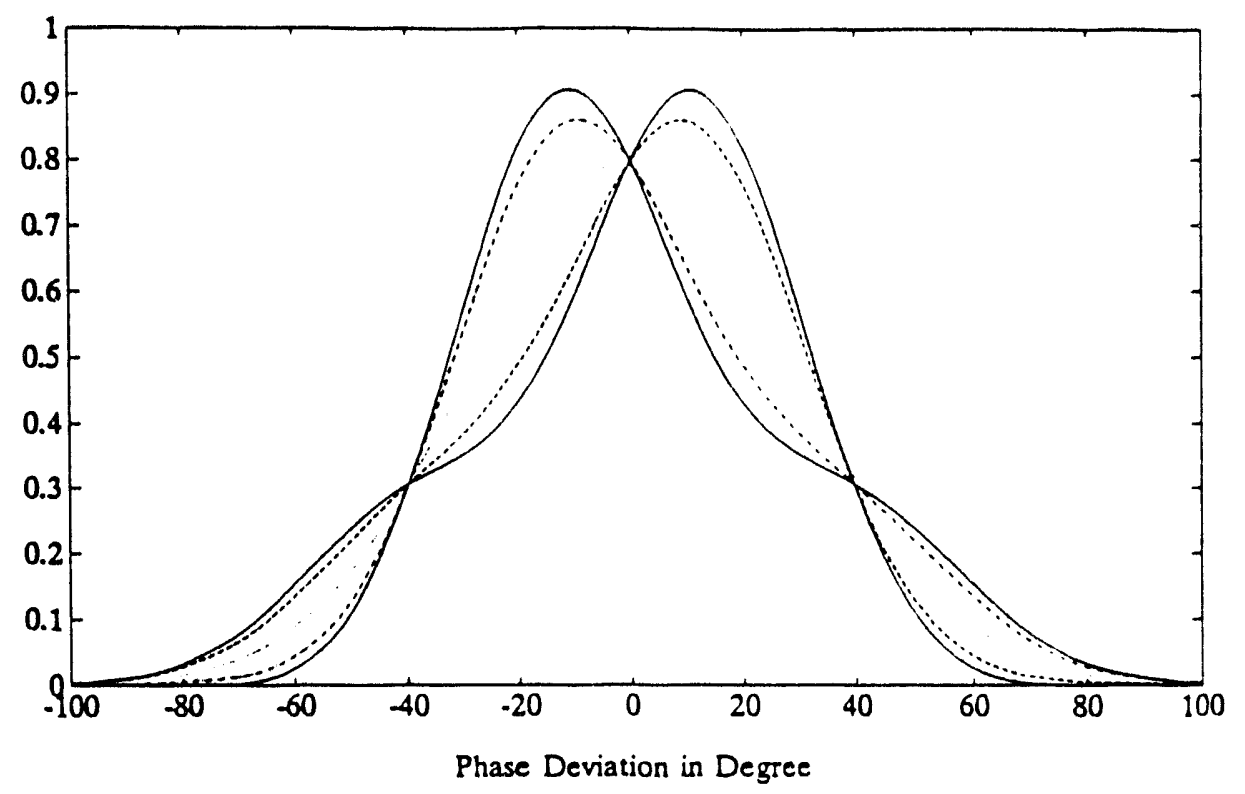

Fig.15. Line Density of Stationary Distribution and 2nd Radial Mode.

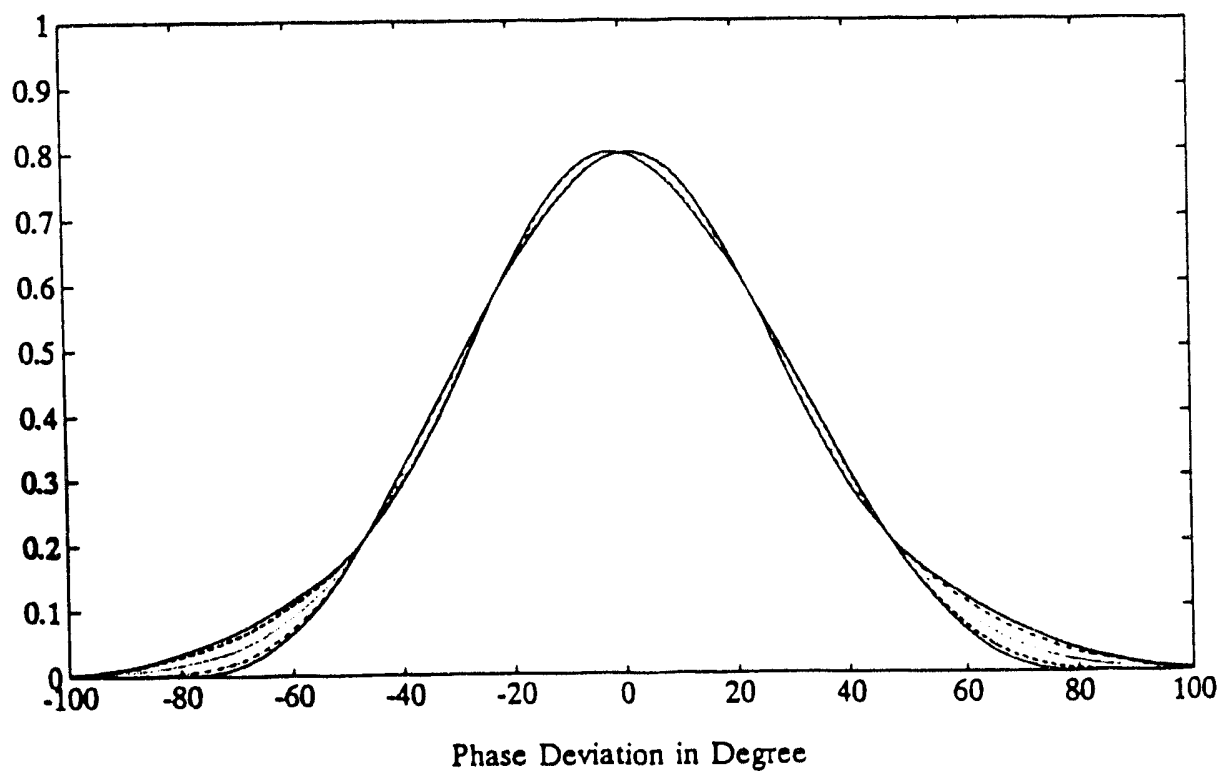

Fig.16. Line Density of Stationary Distribution and 3rd Radial Mode. 


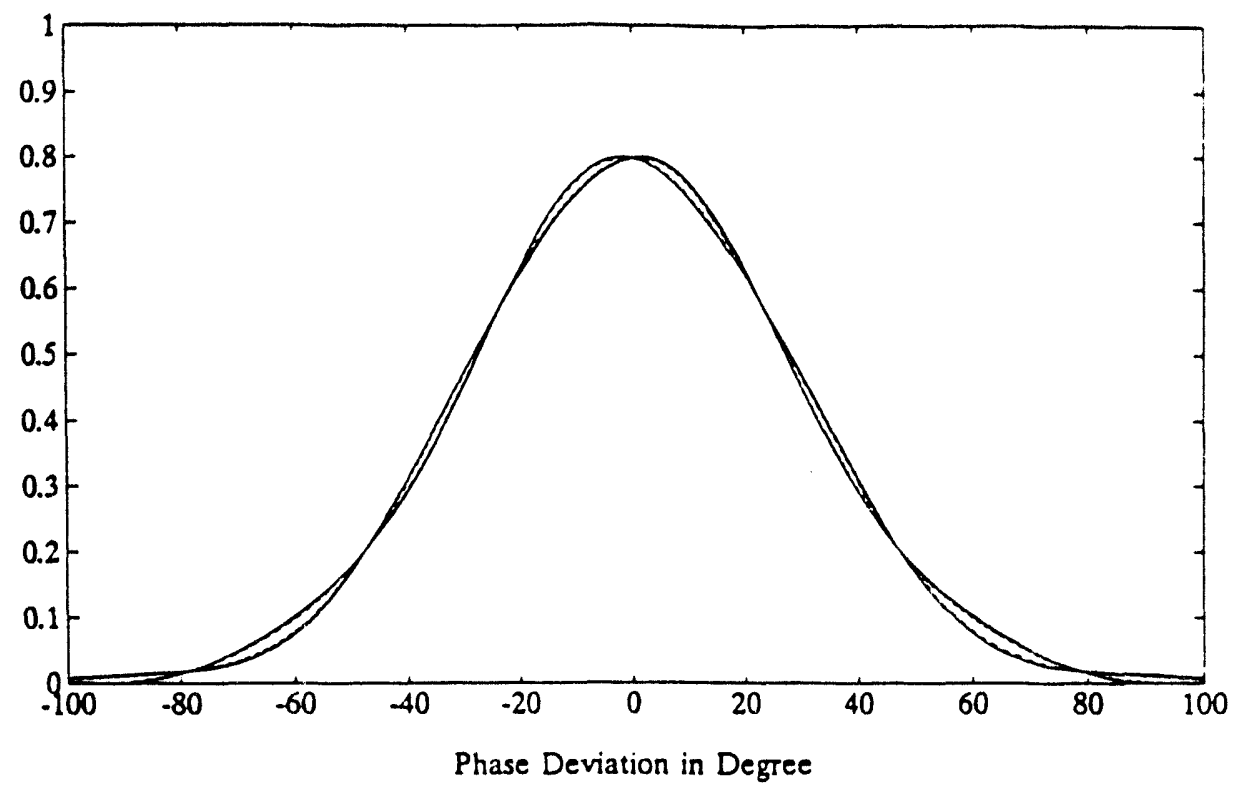

Fig.17. Line Density of Stationary Distribution and 4th Radial Mode.

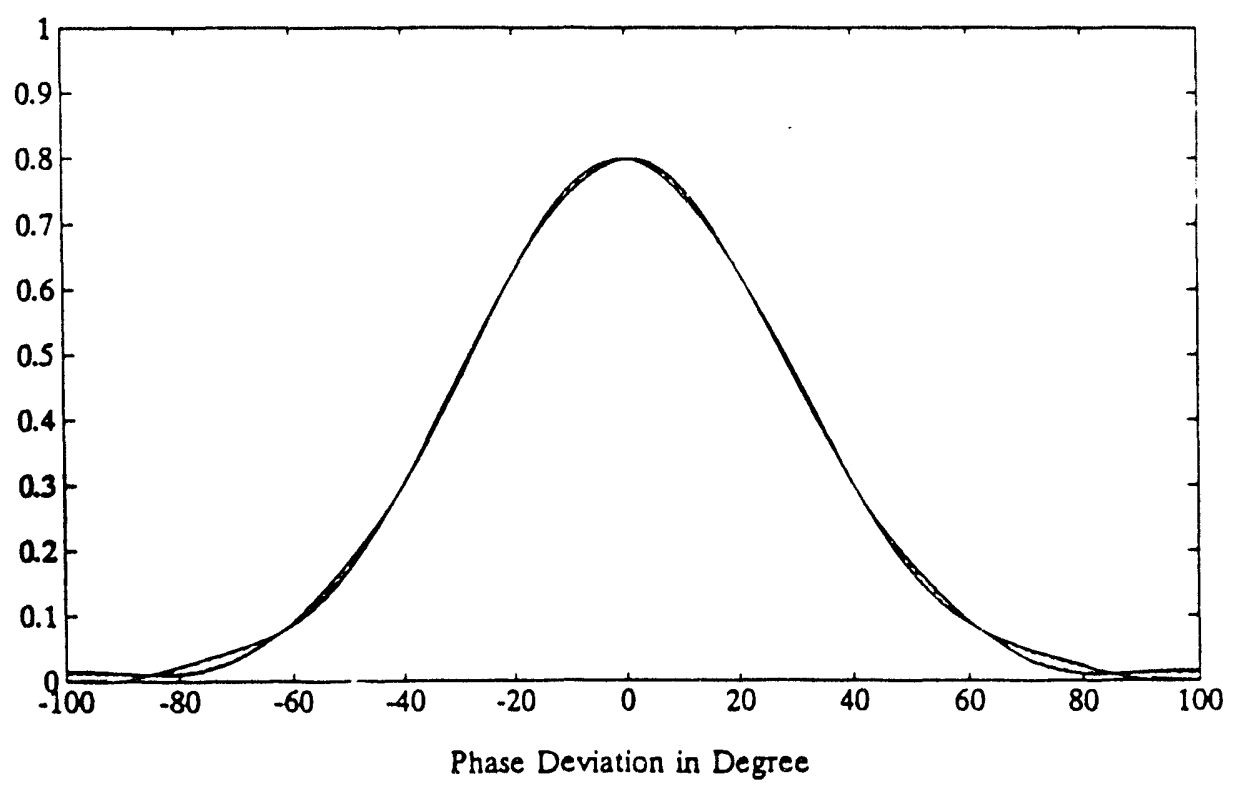

Fig.18. Line Density of Stationary Distribution and 5th Radial Mode. 


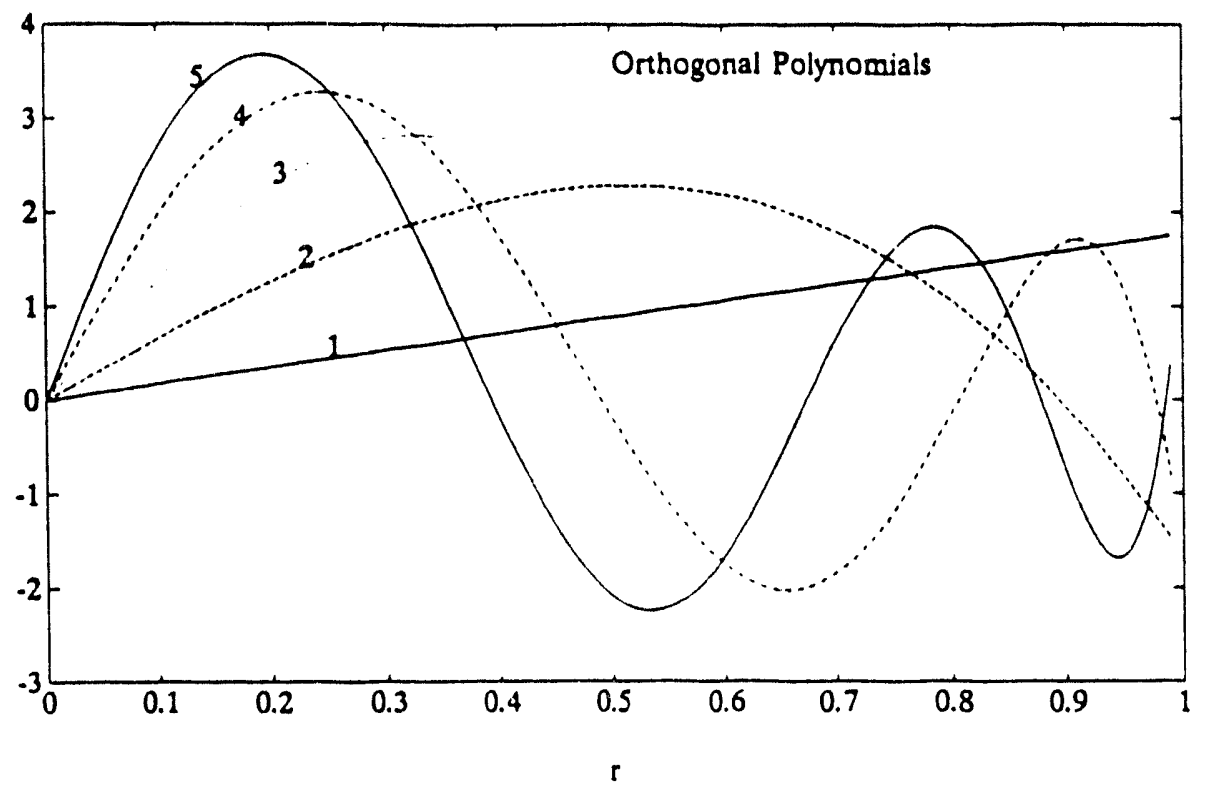

Fig.19. Orthogonal Polynomials of Elliptic Distribution.

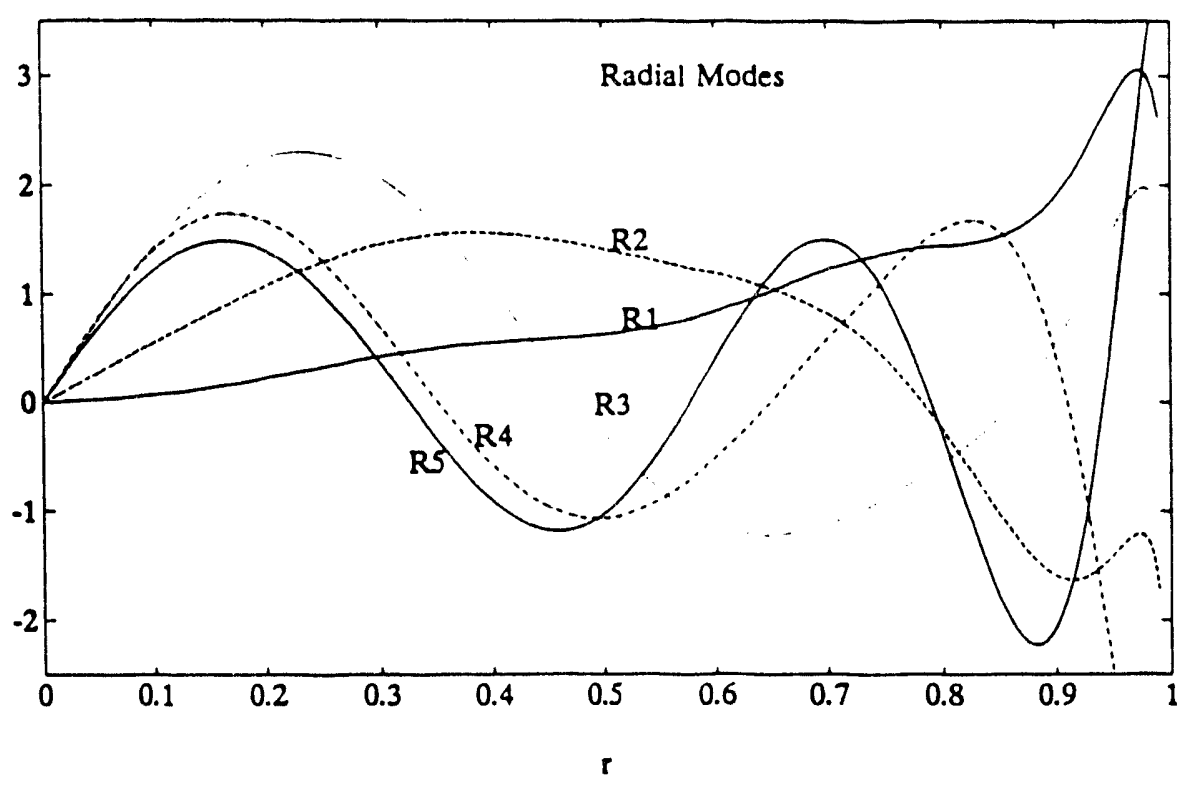

Fig.20. Radial Modes of Elliptic Distribution. 


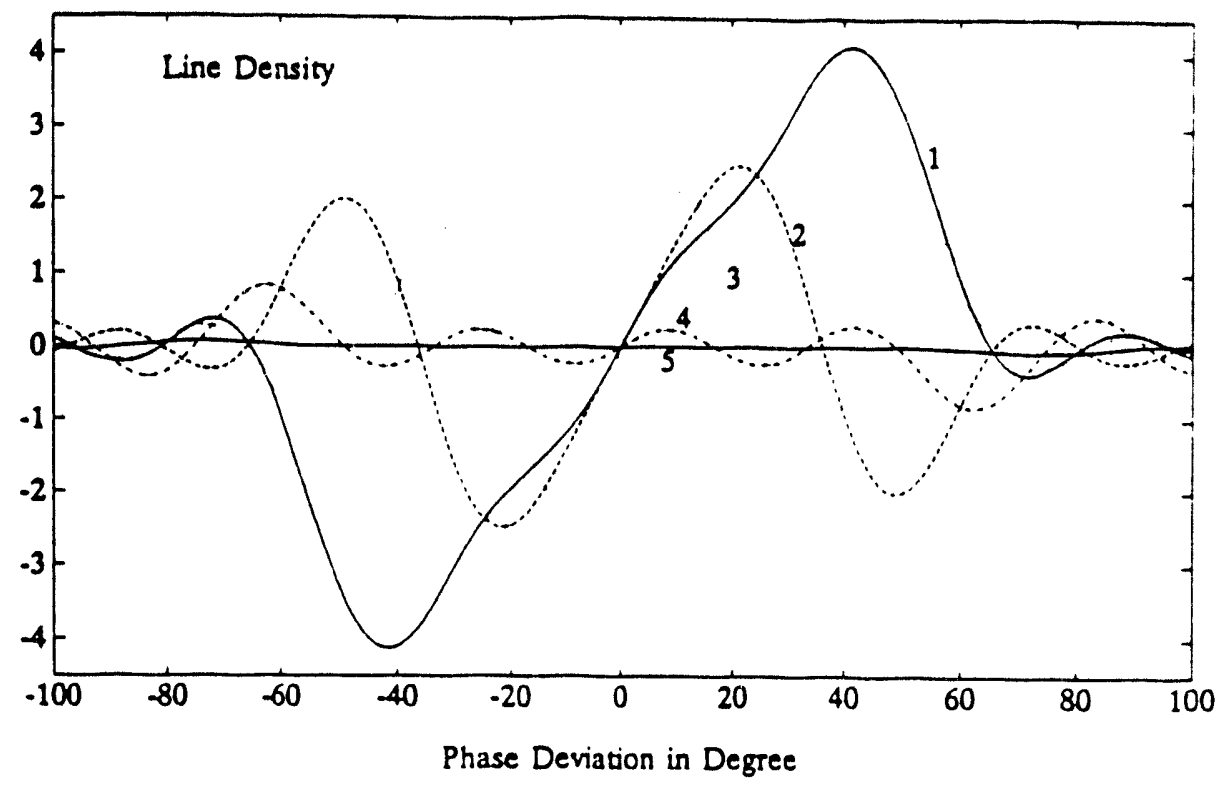

Fig.21. Line Density of Elliptic Distribution.

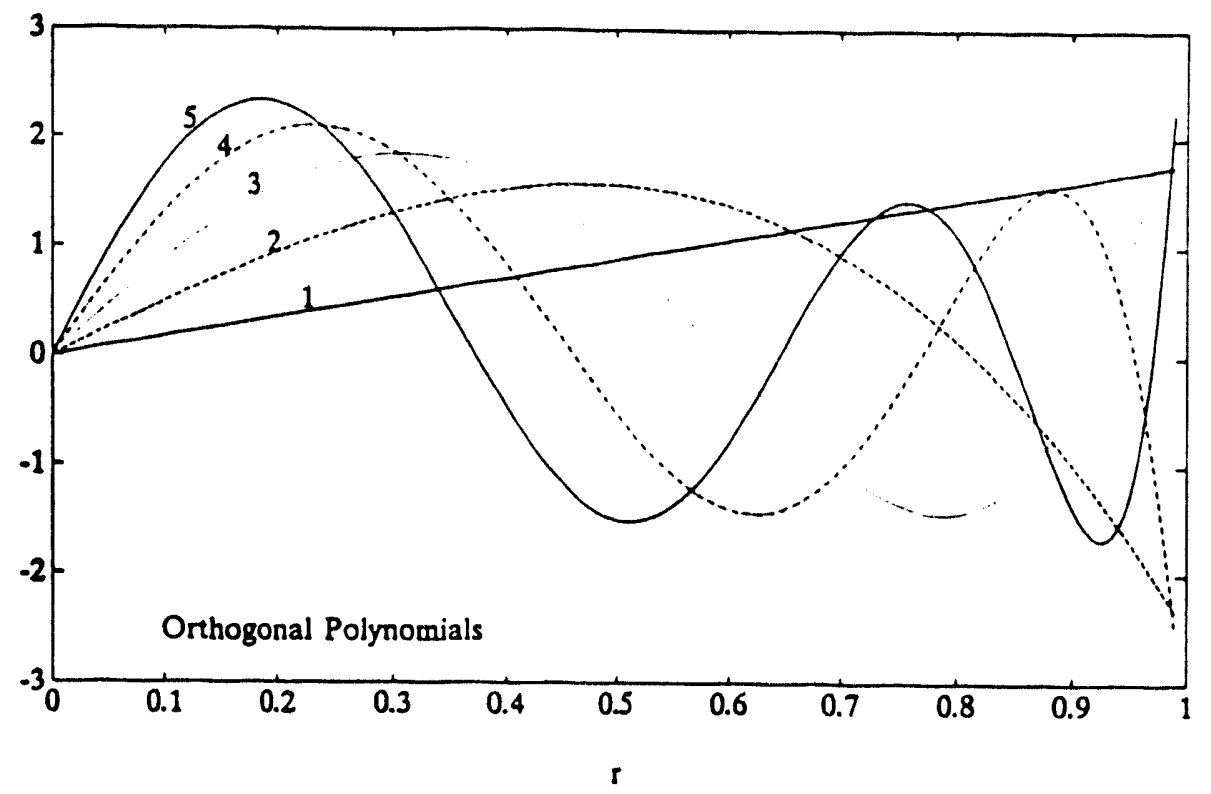

Fig.22. Orthogonal Polynomials of Parabolic Distribution. 


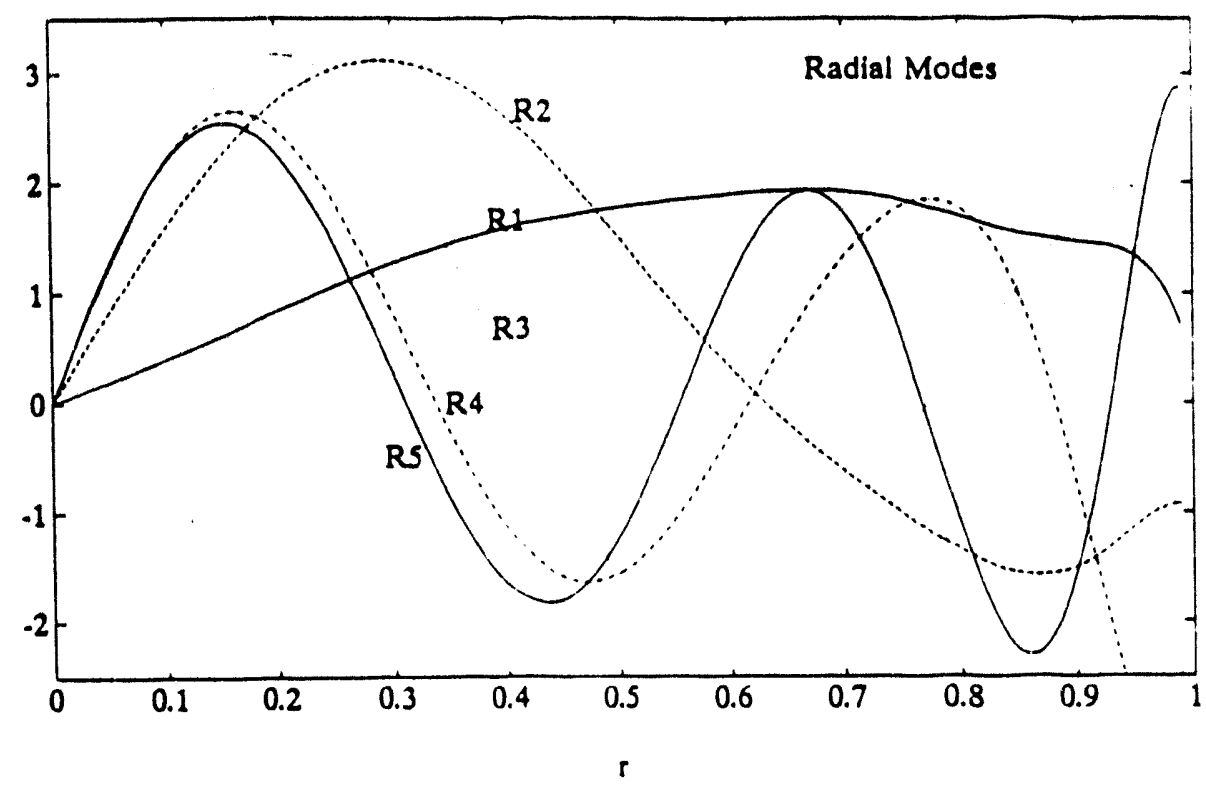

Fig.23. Radial Modes of Parabolic Distribution.

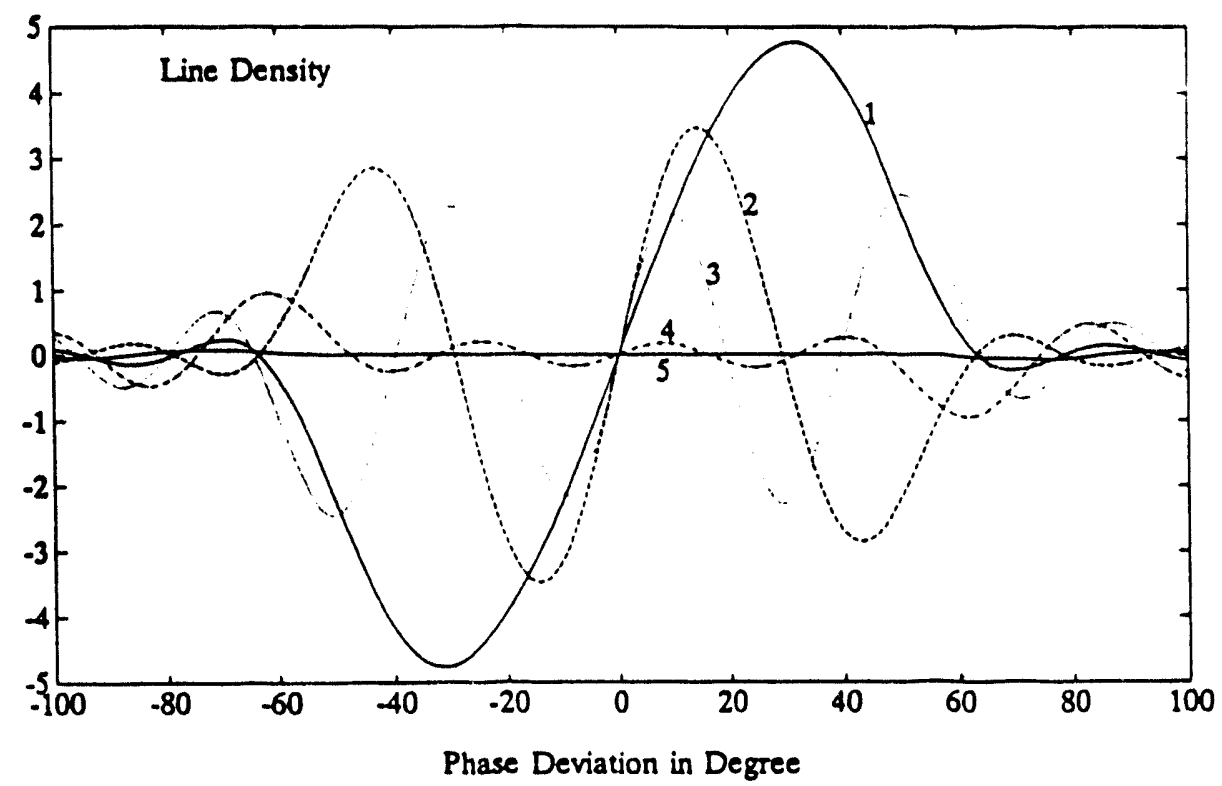

Fig.24. Line Density of Parabolic Distribution. 


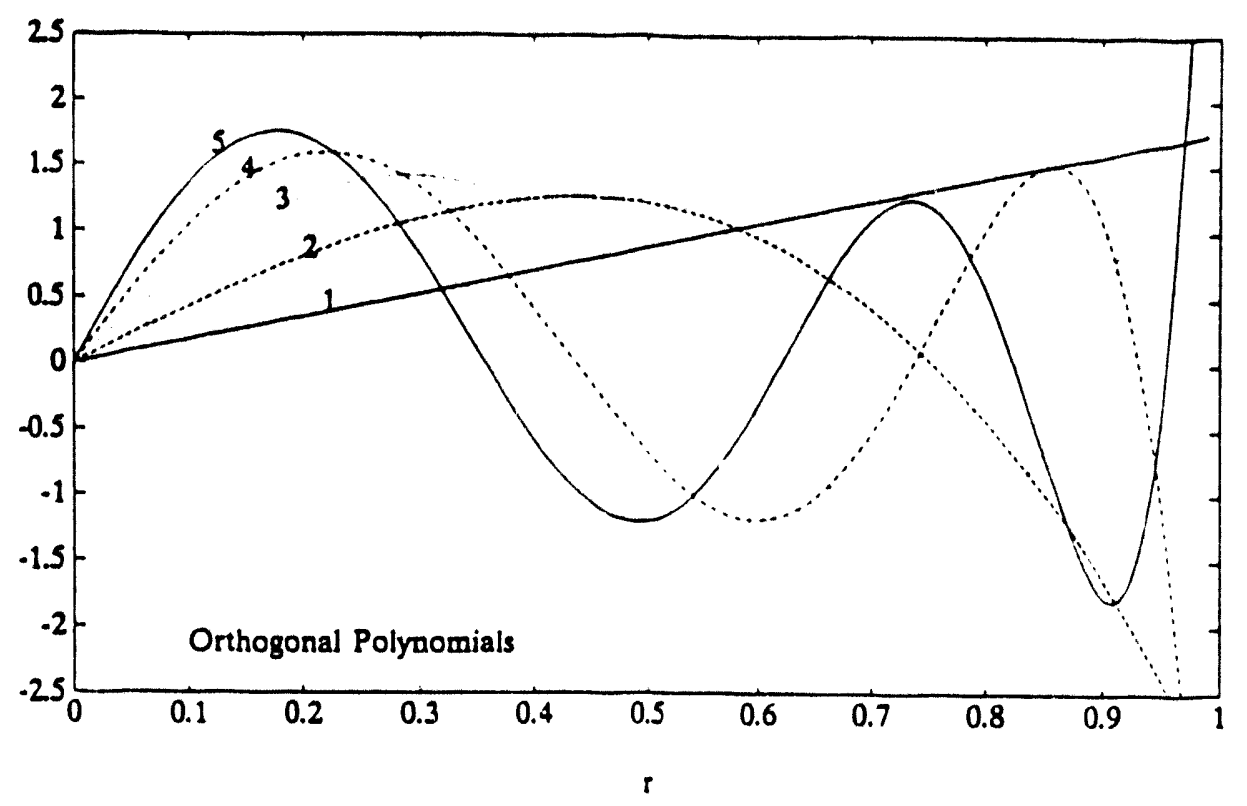

Fig.25. Orthogonal Polynomials of Elliptic Weight Function Distribution.

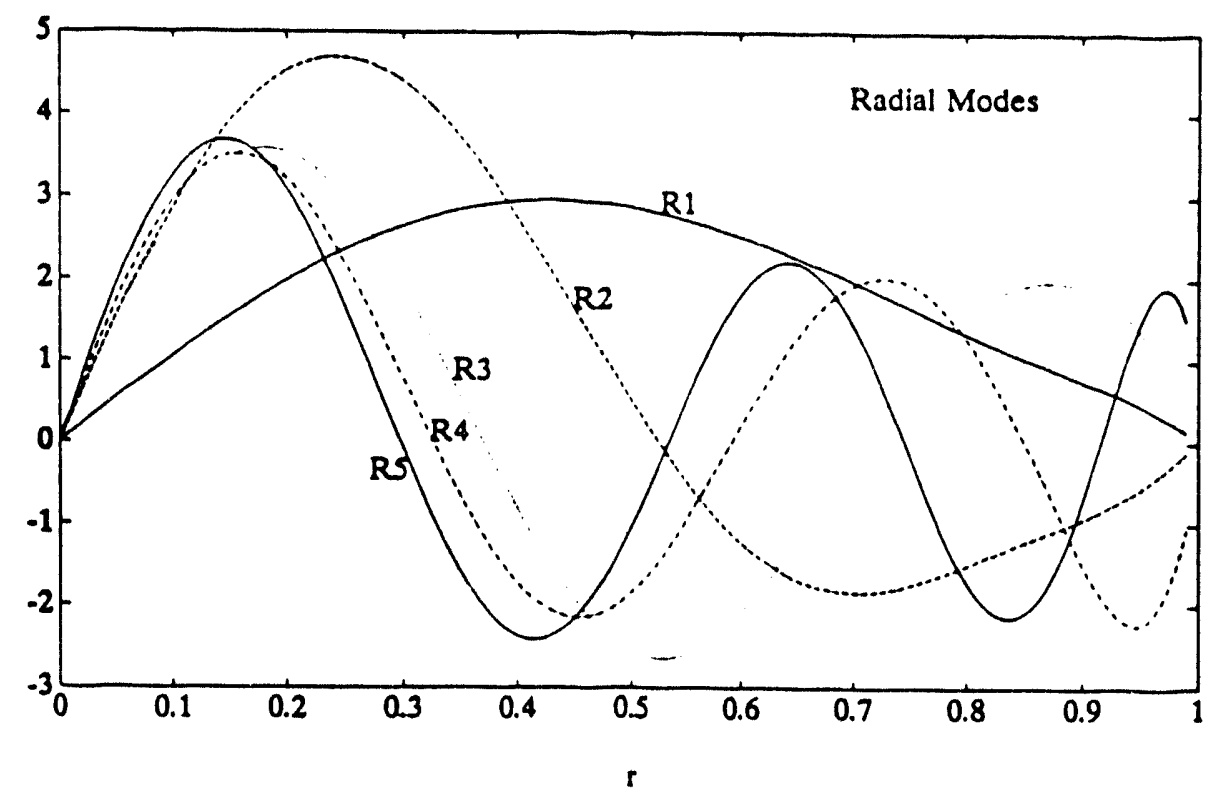

Fig.26. Radial Modes of Elliptic Weight Function Distribution. 


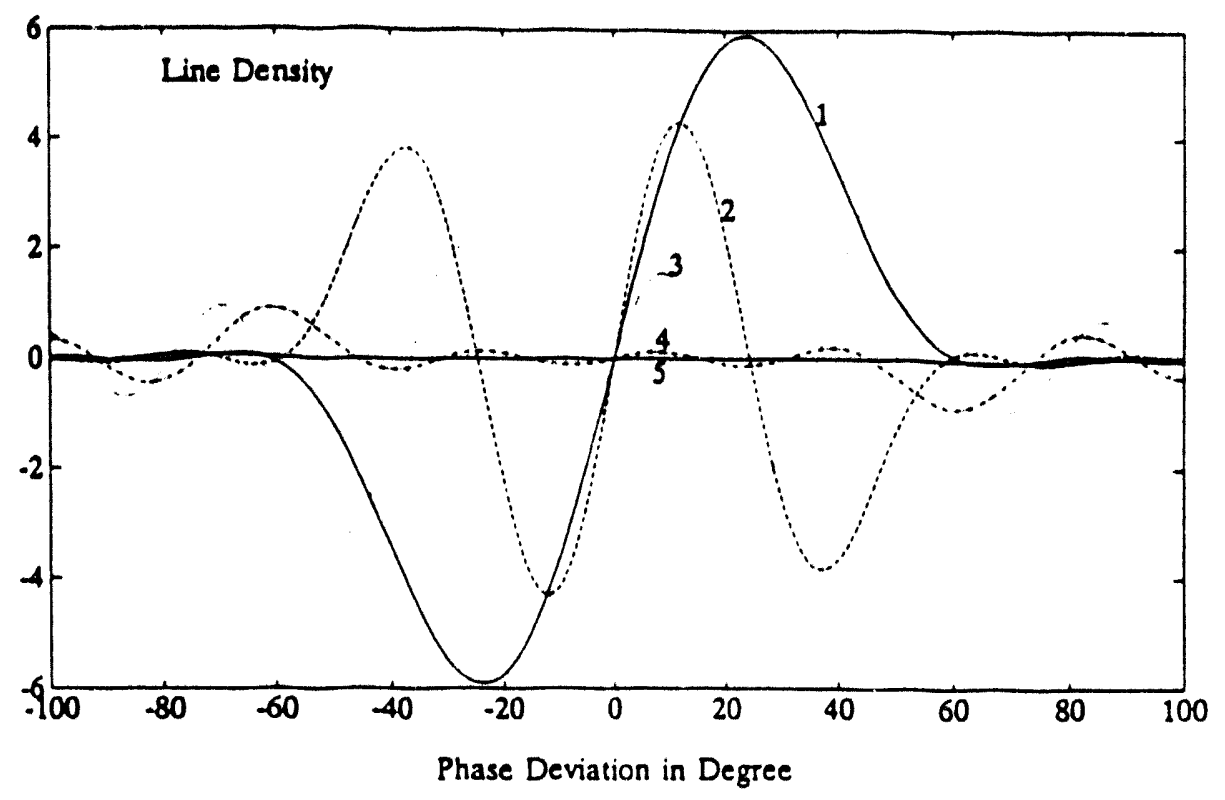

Fig.27. Line Density of Elliptic Weight Function Distribution.

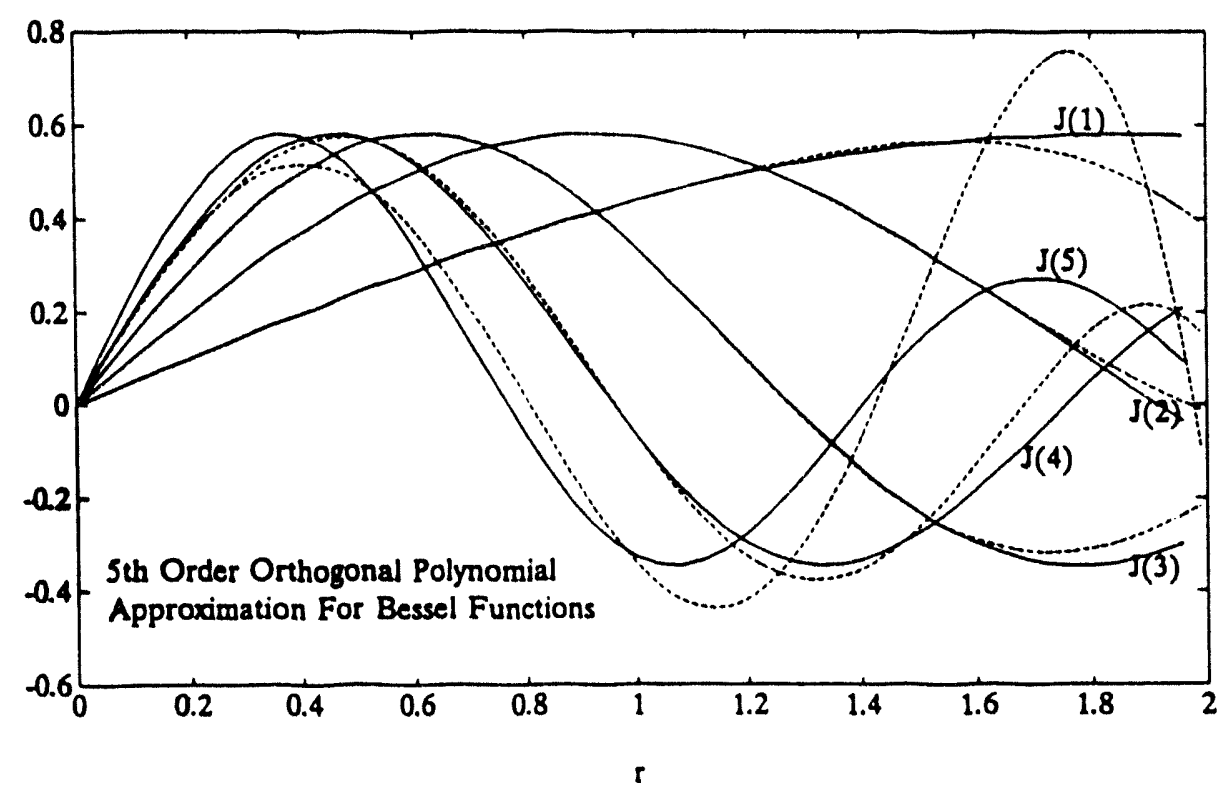

Fig.28. Orthogonal Polynomial Approximation for Bessel Functions. 

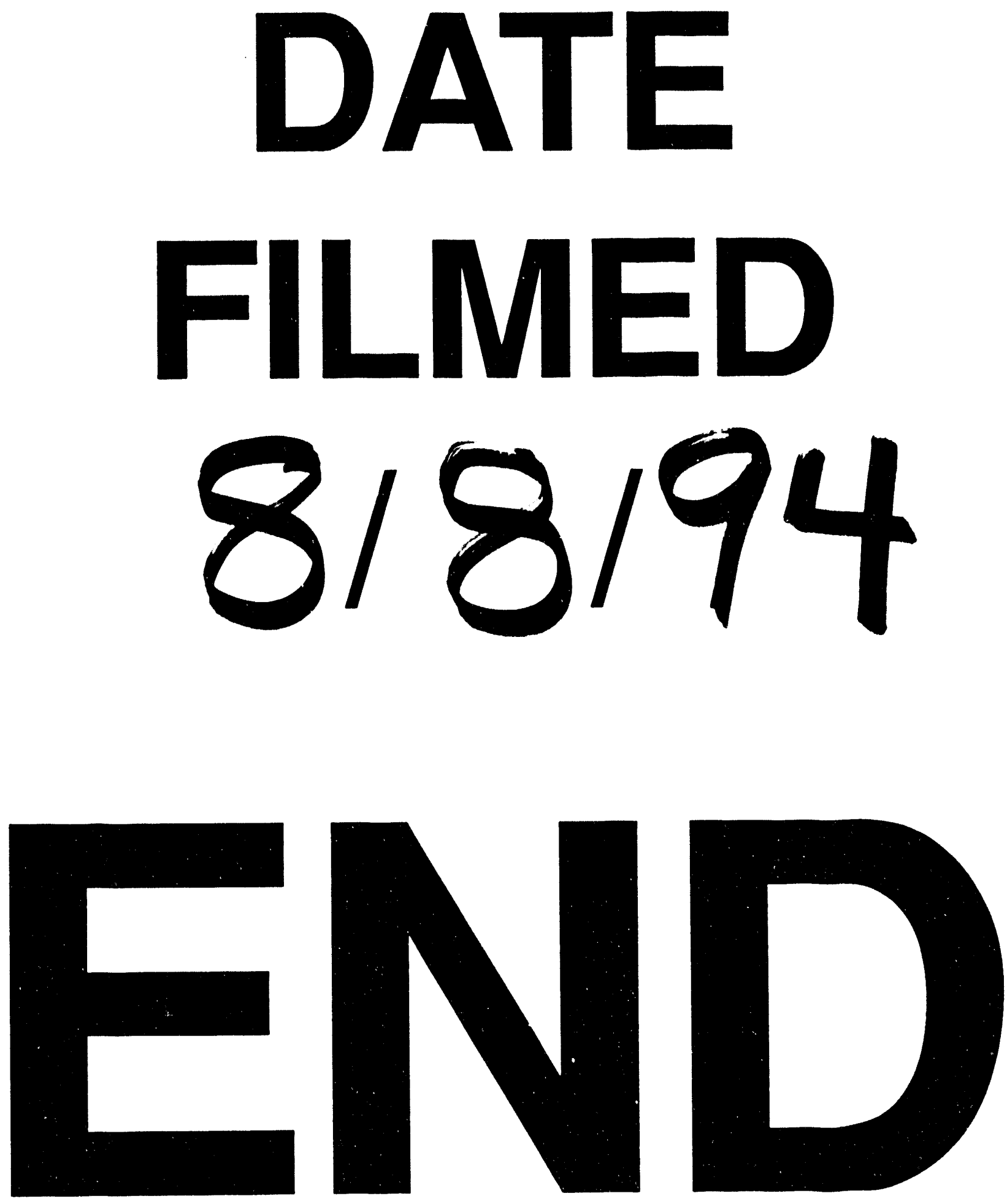
